\title{
To the theory of the electric activity of He II induced by waves of first and second sounds
}

\author{
Maksim D. Tomchenkd * \\ Bogolyubov Institute for Theoretical Physics, 14-b Metrologicheskaya Street, Kiev 03680, Ukraine
}

(Dated: December 5, 2018)

\begin{abstract}
An approximate microscopic model is proposed for the explanation of the electric signal $\triangle U \approx$ $k_{B} \triangle T / 2 e$ observed by A. S. Rybalko in He II in the experiments with standing half-wave of second sound. The model is based on the idea, due to Gutlyanskii, of the one-directional polarization of ${ }^{4} \mathrm{He}$ atoms located at the electrode surface. The calculated parameters of the electric signal are in approximate agreement with the experimental ones. It is also predicted that a standing half-wave of first sound should induce a variable signal with amplitude $\Delta U \sim \triangle p /(|e| n) \sim 3 \cdot 10^{-5} \triangle p \mathrm{~V} /$ atm at the electrode. It is shown also that the dependence of the polarizability of helium on temperature, $A(T)$, can be explained if the tidal polarization of atoms is taken into account. A possibility of the existence of the "dry" friction in He II at temperatures $T \lesssim 0.5 \div 1 \mathrm{~K}$ is discussed.
\end{abstract}

PACS numbers: $67.25 . \mathrm{dg}, 34.35 .+\mathrm{a}$

\section{INTRODUCTION}

In the experiment of Rybalko $\frac{1}{\text {, }}$ a standing half-wave of second sound was generated in He II placed in a metal resonator. In this case, a variable potential difference between the electrode on the resonator end (inside the resonator) and the ground was registered. This electric signal oscillated with the frequency of the second sound and the amplitude $\Delta U \approx k_{B} \Delta T / 2 e$, which was independent of the resonator sizes and the temperature, in the region $T=1.4 \div 1.8 \mathrm{~K}$. Here, $e$ is the electron charge, and $\Delta T$ is the amplitude of temperature oscillations in the secondsound wave. This effect was rather unexpected since free ${ }^{4} \mathrm{He}$ atoms are neutral and do not possess electric dipole or higher multipole moments. After six years since the first communication of the effect, it remains unexplained, in our opinion, in spite of a number of attempts $2-12$. The present work is aimed at a microscopic explanation of the Rybalko effect.

He II is electrically active apparently due to the tidal polarization of helium atoms ${ }^{6.13}$ caused by interaction with neighbors ${ }^{14}-16$. As shown in Ref. 7, the tidal mechanism causes bulk polarization of He II by a wave of second sound. In this case, however, the electric signal is 1-2 orders of magnitude below the registered one and strongly depends on the temperature and resonator size, which is not observed in experiments. It is important that the signal should strongly depend on the resonator size for any bulk mechanism of "spontaneous" polarization of $\mathrm{He} \mathrm{II}^{6,7}$. In the recent works ${ }^{11,12}$, some bulk models without any dependence of the resulting signal on the resonator size were proposed. But we do not agree with the way of the determination of the electric field there. Below (see Appendix A), we discuss some methods of calculation of the field for the given problem.

The idea that the experimental electric signal is related to the polarization of ${ }^{4} \mathrm{He}$ atoms located at the electrode surface was put forward in Ref. 8 and, later on, in Refs. 9, 10. The surface atoms are polarized in the di- rection perpendicular to the surface because of the asymmetry of forces acting on them in this direction: helium atoms are on the one side, and atoms and electrons of metal are on the other side. However, only several layers of helium atoms are strongly polarized, and the electric signal from them may be too weak (it was not calculated in Refs. 8-10). In Refs. 9, 10, a general analysis within the model of a gas consisting of small electron-hole pairs was performed. However, such an approach is indirect and can be used at most only qualitatively because a ${ }^{4} \mathrm{He}$ atom possesses more complicated structure in comparison with an electron-hole pair, and the mass of the atomic nucleus is much greater than the electron mass.

For purely geometric reasons, one can think of the following four sources of the signal:

1. the bulk polarization of He II,

2. the near-surface polarization - from regions in helium at distances from the electrode much larger than the interatomic distance but much smaller than the resonator size,

3. the surface polarization - from several layers of ${ }^{4} \mathrm{He}$ atoms at the electrode surface, and

4. the thermo-emf in the electrode.

The bulk signal is excluded because the bulk polarization is modulated by the wave of second sound. In this case, the larger is the wavelength $\lambda_{2}$ of second sound, the greater is the distance at which the temperature difference is "smeared". Consequently, the signal must strongly depend ${ }^{6}$ on $\lambda_{2}$ and, therefore, on the resonator length $L_{r}$ as well, because of the relation $L_{r}=\lambda_{2} / 2$.

The near-surface polarization hypothetically can be related to some motion of quasiparticles at distances of hundreds or thousands of atomic layers from the electrode. But we are unable to indicate a specific physical mechanism that could cause such a motion under the conditions of the experiment under discussion. Possibly, 
this source of polarization can only be a part of the bulk polarization.

The thermo-emf contributes undoubtedly, but this contribution is apparently small. Moreover, it must strongly depend on the electrode material for both the contact thermo-emf and the thermo-emf driven by the temperature gradient inside the electrode, while the experiment showed that the signal is identical for three different electrodes.

Therefore, the polarization of helium at the electrode surface seems to be the most probable effect. It is this option that will be studied in detail in this paper. In addition, we are going to calculate the parameters of the electric signal induced by a standing half-wave of the first sound.

The main results of the work, except for Sec. III, V and Appendix B, were published in Ref. 17. Part of the results of Sec. II and IV was published also in Ref. 18. The content of Sec. III was published in Ref. 19 .

\section{POLARIZATION OF A ${ }^{4} \mathrm{HE}$ ATOM LOCATED AT THE BOUNDARY BETWEEN HE II AND METAL}

In this section, we calculate the dipole moment (DM) of a ${ }^{4} \mathrm{He}$ atom located at a plane boundary between He II and metal. Let the metal and helium occupy the halfspaces $z<0$ and $z>0$, respectively. The $z$ axis is directed into helium normally to the metal surface. The DM of a single nonpolar atom at a distance $z_{0}$ from the plane surface of a metal was calculated in Ref. 20:

$$
\begin{gathered}
\mathbf{d}_{\mathrm{mir}}=D_{4}|e| \frac{a_{B}^{5}}{z_{0}^{4}} \mathbf{i}_{z}, \\
D_{4} \approx \frac{3}{8} \frac{e^{2}}{\hbar a_{B}} \sum_{n} \frac{\left\langle z_{n}^{4}+z_{n}^{2} x_{n}^{2}\right\rangle}{a_{B}^{4}} \frac{a}{b \omega_{a}\left(\omega_{a}+b\right)} .
\end{gathered}
$$

Here, the summation is performed over the electrons of an atom (for a ${ }^{4} \mathrm{He}$ atom, $n=1,2$ ), $a=b^{2}=\omega_{\mathrm{pl}}^{2} / 2$, $\omega_{\mathrm{pl}}$ is the plasma frequency of a metal, $\omega_{a}=\Delta E / \hbar$ is the effective excitation frequency of the atom, $x_{n}$ and $z_{n}$ are the coordinates of the $n^{\text {th }}$ electron of the atom, $a_{B}=\hbar^{2} /\left(m e^{2}\right)=0.529 \AA$, and the averaging is performed over the ground state of the atom. Physically, the DM (11) arises due to interaction of the atom with its "image" in a metal "mirror," hence the notation $\mathbf{d}_{\text {mir }}$. The image is not an exact mirror image but represents a small perturbation of the distribution of charges in a metal at large distances from the position of the exact image. However, this distribution is equivalent to an exact mirror image in its action, which follows also from results in Ref. 21. That work presents the study of the polarization of an atom by a dielectric with the use of a more transparent method - the DM was calculated as the sum of DMs induced by each of the dielectric atoms with regard for the response; the result was identical to that in Ref. 20 .

For a ${ }^{4} \mathrm{He}$ atom, the quantity $D_{4}$ in Eq. (2) can be presented in the form

$$
D_{4} \approx \frac{2 R y}{5 \triangle E} \frac{\hbar \omega_{\mathrm{pl}}}{\hbar \omega_{\mathrm{pl}}+\sqrt{2} \triangle E}\left\langle\frac{r^{4}}{a_{B}^{4}}\right\rangle,
$$

where $R y=e^{2} / 2 a_{B}=13.6 \mathrm{eV}$, and $\triangle E$ is the mean excitation energy of a ${ }^{4} \mathrm{He}$ atom which is close ${ }^{16}$ to the ionization energy ${ }^{22} \Delta E_{\text {ion }} \approx 24.58 \mathrm{eV}$. For the simplest one-parameter wave function $\Psi_{0}$ of the ground state of a ${ }^{4} \mathrm{He}$ atom, we have $\left\langle r^{4} / a_{B}^{4}\right\rangle \approx 2.775$, whereas for the 80parameter $^{23} \Psi_{0}$ close to the exact one, $\left\langle r^{4} / a_{B}^{4}\right\rangle \approx 3.973$ (see Ref. 16). The last value will be used below.

We now consider a ${ }^{4} \mathrm{He}$ atom located in the first layer of helium near the plane surface of the metal. This atom has polarization $\mathbf{d}_{\mathrm{h}}$, induced by all other helium atoms. It was shown in Refs. 14,15 that two interacting ${ }^{4} \mathrm{He}$ atoms induce the following DM on each other:

$$
\mathbf{d}=-D_{7}|e| \frac{a_{B}^{8}}{R^{7}} \mathbf{n}
$$

where $\mathbf{n}=\mathbf{R} / R$ is the unit vector along the direction to the neighboring atom, and $D_{7} \approx 18.4$. In Ref. 16 , a similar formula with $D_{7} \approx 25.2 \pm 2$ was obtained using a simpler method. Taking into account both results, we assume

$$
D_{7} \approx 23 \pm 5
$$

We note that equations (11) and (4) are obtained by neglecting the exchange interaction and the higher corrections (with larger degrees of $z_{0}$ or $R$ in the denominator). Both approximations are valid at $z_{0}, R \gg a_{B}$. For He II, we have $z_{0}, R \gtrsim 3 \AA$, which justifies the applicability of (11) and (4).

According to Eq. (15) from Ref. 14 and Eq. (18) from Ref. 16, the quantity $\mathbf{d}$, given by (4), is proportional to the average of the DM operator and to the square of a perturbing potential (equal to the difference of the total Hamiltonian of two interacting ${ }^{4} \mathrm{He}$ atoms and the Hamiltonians of free atoms, according to Eq. (10) from Ref. 6; this is the sum of the Coulomb potentials). In this case, the exchange interaction is dropped in Refs. 14, 16, which is justified for the interatomic distances $\gtrsim 3 \AA$ under consideration. Let us calculate $\mathbf{d}_{\mathrm{h}}$. The resulting formula for $\mathbf{d}_{\mathrm{h}}$ will include the square of the total perturbing potential, equal to the sum of the perturbing potentials from each atom. The square of the total potential is given by the sum of the squares of one-atom potentials (this gives the sum of DMs induced by individual atoms) and the sum of cross terms, which also give a nonzero contribution to the total DM $\mathbf{d}_{\mathrm{h}}$. Therefore, the DM induced by the sum of atoms is not reduced to a sum of DMs induced by each atom separately. We, however, neglect the corrections from cross terms, which represent three-particle corrections and are usually neglected in many-particle 
problems. Then the polarization $\mathbf{d}_{\mathrm{h}}$ from the collection of helium atoms is equal to the sum of the polarizations $\mathbf{d}_{\mathrm{h} j}$ (4) from separate atoms:

$$
\mathbf{d}_{\mathrm{h}} \approx \sum_{j} \mathbf{d}_{\mathrm{h} j},
$$

and the total DM of a ${ }^{4} \mathrm{He}$ atom located at the metal surface is equal to

$$
\mathbf{d}_{1} \approx \mathbf{d}_{\mathrm{h}}+\mathbf{d}_{\text {mir }} .
$$

The cross terms renormalizes $\mathbf{d}_{\mathrm{h}}$ at most by $20-30 \%$, most likely, as compared with (6).

In view of (4) and (5), the sum (6) can be written in the form

$$
\mathbf{d}_{\mathrm{h}} \approx-d_{0} \int_{0}^{2 \pi} d \phi \int_{0}^{\pi / 2} d \theta \int_{0}^{\infty} r^{2} d r \frac{g(\mathbf{r}) \bar{R}_{0}^{7}}{\bar{R}^{3} r^{7}} \frac{\mathbf{r}}{r} \equiv-\mathbf{i}_{z} \frac{d_{0} S_{7}}{4} \frac{n}{n_{0}},
$$

where

$$
\begin{gathered}
d_{0}=D_{7}|e| a_{B}^{8} / \bar{R}_{0}^{7}=3.56 \cdot 10^{-5}|e| a_{B}, \\
S_{7}=\int_{\Omega_{2}=4 \pi} n_{0} g(\mathbf{r}) \frac{\bar{R}_{0}^{7}}{r^{7}} d \mathbf{r},
\end{gathered}
$$

$n(T, p)=\bar{R}^{-3}(T, p)$ is the concentration of He II, $n_{0}=$ $n(T=1 K, p=\mathrm{svp})=\bar{R}_{0}^{-3}, \bar{R}_{0}=3.578 \AA$, and $g(\mathbf{r})$ is the two-point correlation function, i.e., the probability to find a helium atom at the displacement $\mathbf{r}$ from another helium atom. If the density of helium is constant near the metal, then helium as the simple liquid is isotropic, and $g(\mathbf{r}) \equiv g(r)$. In this case, $n \approx n_{0}$ and $S_{7}=S_{7}\left(n_{0}\right) \approx 14.9$ (see Ref. 7). At the saturation vapor pressure (svp), we obtain the polarization of the first layer of helium at the metal surface:

$$
\mathbf{d}_{\mathrm{h}} \approx-\mathbf{i}_{z} \cdot 1.32 \cdot 10^{-4}|e| a_{B} .
$$

The polarization of the next layers is then determined by equation (29) which is derived below.

\section{PROPERTIES OF HE II NEAR THE METAL SURFACE AND A POSSIBILITY OF THE "DRY" FRICTION}

To calculate $\mathbf{d}_{\mathrm{h}}(\mathbf{8})$, (10), it is necessary to know the state of helium at the metal surface, namely the density profile $\rho(T, p)$ and the function $g(\mathbf{r})$. This state depends on the helium atom- metal potential, and we must else consider the exhaustion of the superfluid (SF) component near the wall.

Depending on the interaction potential between the metal and a ${ }^{4} \mathrm{He}$ atom, helium near the metal surface can be in four different states 24,25 . For the weakest potential (Cs), helium does not wet the metal, and the first layer of helium near the metal is liquid. For stronger potentials $(\mathrm{Rb}, \mathrm{Na}, \mathrm{K}, \mathrm{Li})$, some wettability is present, but the first layer is still liquid. Most metals $(\mathrm{Cu}, \mathrm{Al}$, $\mathrm{Au}$ and others) have stronger interaction potential, making the first layer of helium solid, and the second one liquid. Finally, at the strongest possible potentials, two layers can become solid. The authors of Ref. 25 believe that this happens for $\mathrm{Au}$. However, the potential for $\mathrm{Au}$ (see Ref. 26) at the distance of two atomic layers is approximately equal to the interaction potential of two ${ }^{4} \mathrm{He}$ atoms and cannot compress helium up to solidification. Therefore, we assume that only one layer of helium is solid for Au.

To know the dependence $T(z)$ for helium at a wall, it is necessary to understand the properties of the SFcomponent near the wall. In the presence of the wetting, helium atoms adhere to the wall, but $\mathbf{v}_{s}$ cannot continuously increase, as the distance from the wall increases. In this connection, V.L. Ginzburg advanced the assumption 27 that $\mathbf{v}_{s}$ has a discontinuity near the wall, and, therefore, the "dry" friction must be observed in helium-II. However, the experiment gave no evidence of such a friction ${ }^{28}$. This implies ${ }^{29}$ that $\rho_{s}=0$ on the metal surface. To the best of our knowledge, the microscopic reason for such an exhaustion of $\rho_{s}$ is not clarified. Since $\rho_{s}$ is equal to $\rho-\rho_{n}$ by definition, the exhaustion of $\rho_{s}$ on the wall can be caused by the behavior of $\rho$ (i.e., that of atoms) or by the behavior of $\rho_{n}$ (i.e., that of quasiparticles). The first seems improbable $-\rho$ ensures $\rho_{s}=0$ either due to $\rho=0$ (but the exact zero cannot be reached, since the wall does not represent the infinitely high energy barrier) or because of the exact equality $\rho=\rho_{n}$, whose validity is improbable due to the properties of atoms $(\rho)$ - there is no reason for atoms to be rearranged so that the relation $\rho=\rho_{n}$ be satisfied namely on the wall. It would be so that $\rho$ is very close to zero on the wall (i.e. $\rho_{s}$ is almost zero), but the dry friction arising in this case would be too low for the experimental registration. But, in this case, $\rho_{s}$ should be close to zero only at the wall at the distance which is significantly less than the mean interatomic one (indeed, nothing hampers atoms to approach one another so closely). However, the experiment ${ }^{30,31}$ shows that $\rho_{s}$ is close to zero at significantly larger distances from the wall equal approximately to two atomic layers. This is namely the effective radius of a roton ${ }^{32}$. Hence, we may conclude that, most likely, $\rho_{s}=0$ due to the properties of quasiparticles. In other words, there is a certain reason for the concentration of quasiparticles to be maximal on the wall and for the condition of the $\lambda$-transition, $\rho_{s}=0$, to be realized. Below, we will study this possibility.

The following simple mechanism is possible. The microscopic calculation ${ }^{33,34}$ and the experiment ${ }^{35}$ imply that the energy $\Delta_{2 D}$ of a surface (2D) roton is approximately by $2 \mathrm{~K}$ less than the energy of a bulk (3D) roton. It is seen from the spectrum of $2 \mathrm{D}$ - and $3 \mathrm{D}$-rotons $33-35$ that, near the wall, 3D-roton can create a 2D-roton with the emission of a 3D-phonon (a) or the inverse process (b) 
is possible. The creation of a 3D-roton and a 3D-phonon by a $2 \mathrm{D}$-roton and the inverse process are forbidden by the energy conservation law. The creation of a 2D-roton must dominate over its absorption, since the former is determined only by the probability of the process itself, whereas the probability of the absorption is proportional else to the concentration of phonons with the required momentum. In other words, a 2D-roton can fuse with a 3D-phonon only if such a phonon will be near, whereas a 3D-roton can decay at once. At $T \gtrsim 1 \mathrm{~K}$, the number of rotons is great, and the rotons approaching the wall must decay into a 3D-phonon and a 2D-roton, until the maximally possible concentration of $2 \mathrm{D}$-rotons will be attained. In other words, $\rho_{n}$ becomes equal to $\rho$, and $\rho_{s}$ becomes zero on the surface, which is observed in experiments. At very low $T \lesssim 0.1 \mathrm{~K}$, the number of rotons less than the number of phonons by many orders. In this case, if the maximally possible concentration of $2 \mathrm{D}$-rotons on the surface would be conserved as before, then process (b) would dominate, because it is proportional to the large concentrations of 2D-rotons and 3D-phonons, and processes (a) is proportional to a very low concentration of 3D-rotons. Process (b) will continue, until, first, the concentration of 2D-rotons drops to a certain equilibrium one, and, second, the temperature $T$ of the wall becomes much less than $T_{\lambda}$. Since the spectrum of 3D-phonons at low energies coincides with the (theoretical) spectrum of 2D-phonons ${ }^{33.34}$, the temperatures in the bulk and on the surface must be close, i.e., $\rho_{s} \approx \rho$ at the surface. The critical temperature $T_{c}$, at which the exhaustion of $\rho_{s}$ on the wall disappears, is probably near $T_{c} \simeq 0.5 \div 1 \mathrm{~K}$ which is the temperature $T$ of the transition from the dominance of rotons to the dominance of phonons.

We note that as early as 1941 P. L. Kapitsa ${ }^{36}$ observed a jump of $T$ in near-surface layers of helium near a heater. In this case, the heater was supplied by a heat flow. Such a jump was explained theoretically in Ref. 37, but those calculations did not involved surface excitations of helium. The above-presented reasoning shows that a jump of $T$ must be observed in the first several atomic layers of helium near the wall due to surface excitations. Importantly, that in this case we have an equilibrium state without a heat flow, but with the gradient of $T$. The nature of this jump is different from that of the Kapitsa's jump, the latter being related to the high heat conductivity of helium which implies that the equilibrium is established, in the first turn, in bulk and surface helium. The heat exchange with the wall is much more slower. In this case, the heat exchange between surface excitations of helium and the wall is obviously possible. But it should be expected that it is insignificant. Therefore, a small jump of $T$ between the bulk wall and bulk helium should be present even in the absence of a heat supply to the wall or helium, which can be verified experimentally. In this case, it is necessary to take into account that such a jump of $T$ should be present between a heater and helium, as well as between a thermometer and helium.

It is of significance that, at $T \leq T_{c}$, we must observe the "dry" friction, which can be verified in a direct experiment like ${ }^{28}$ or by measuring the temperature dependence of the peak of a surface roton on the temperature 35 . In experiments with the third sound ${ }^{30}$, it was found that the recovery length for $\rho_{s}$ increases with $T$ at $T \gtrsim 1 \mathrm{~K}$ and is constant at $T \lesssim 1 \mathrm{~K}$. These dependencies have no explanation, and it is possible that $T \approx 1 \mathrm{~K}$, at which the character of the dependence varies, is $T_{c}$. In Ref. 28 , the temperature of the experiment was not given, but such experiments are usually carried on at $T>1.2 \mathrm{~K}$. If the dry friction will not be discovered at small $T$ or turn out much less than that estimated in Ref. 27, then $\rho_{s}$ is exhausted on a wall due to a decrease of the total density almost to zero, rather than due to the accumulation of 2D-rotons.

The ideas of this section are considered in more detail in Ref. 19.

\section{ELECTRIC SIGNAL FOR HE II WITH A WAVE OF SECOND SOUND}

Let us calculate the electric signal induced by second sound in He II at electrodes made of metals of four types enumerated in Sec. III.

\section{A. Electrodes with a strong potential}

We consider electrodes made of material that solidifies the first layer of helium near their surface. In the experiment ${ }^{1}$, three electrodes were used: those made of gold, brass (it consists mainly of copper), and ruthenium oxide. For gold and copper, the first layer of helium near the metal is solidified, whereas the second one is liquid with an enhanced density ${ }^{31}$ corresponding to $p \simeq 10-15$ atm. As the distance to the metal increases by several atomic layers, the density decreases to the bulk density.

Consider the first layer of helium atoms at the electrode. This layer is polarized perpendicularly to the electrode surface with the value of polarization given by (7) and (8). To calculate $\mathbf{d}_{\mathrm{h}}$, we must find $S_{7}$ (10). Since the first layer is solid and the following ones are liquid but at a varying pressure, the function $g(\mathbf{r})$ in (10) must be rather complicated. However, about $95 \%$ of the contribution to $S_{7}$ comes from the nearest layer, as is follows from our numerical calculation. Therefore, we can determine $S_{7}$ approximately, taking $g(\mathbf{r})$ to be isotropic and corresponding to the pressure of this layer. For the liquid layer, we take $p \approx 13$ atm. Since ${ }^{7} S_{7} \sim \rho^{4 / 3}$, relation (8) yields $\mathbf{d}_{\mathrm{h}} \sim \rho^{7 / 3} \sim \bar{R}^{-7}$. Therefore, we can determine $\mathbf{d}_{\mathrm{h}}$ approximately, by multiplying (11) by $\left[2 \bar{R}_{0} /\left(\bar{R}_{1}+\bar{R}_{2}\right)\right]^{7} \approx 1.56$, where $\bar{R}_{1}$ and $\bar{R}_{2}$ are the mean interatomic distances in the first and second layer, respectively. Then

$$
\mathbf{d}_{\mathrm{h}} \approx-\mathbf{i}_{z} \cdot 2.06 \cdot 10^{-4}|e| a_{B}
$$


The data on the density and thermal expansion coefficient of helium can be taken from Ref. 38 .

We now calculate the potential induced by the first layer of helium on the electrode. In experiments, the potential difference between the electrode on the internal surface of a resonator and the resonator itself (insulated from each other), whose external surface was grounded, was registered. In other words, it is the potential difference between the electrode and a point at infinity. The dipole layer (DL) in helium near the internal surface of the resonator induces DL of "images" on this surface. As a result, the potential on the internal surface of the electrode that is induced by both DLs is equal to zero at all points. It is difficult to find the total potential of the electrode with regard for all contributions. But we need only the variable part of the potential difference that can be easily determined. We use the formula

$$
\triangle \varphi=-\int \mathbf{E d s}
$$

and draw a contour from a point on the electrode surface to infinity. In calculations of the surface contribution, we take only a part of the contour near the electrode into account. The next part up to the resonator wall gives the bulk potential difference, and the following part with intersections of the resonator, vessel, etc. can be omitted, since this part is invariable in time, whereas we are interesting in the variable part of the potential created by oscillations in a wave of second sound. From the relation $\mathbf{E}+4 \pi \mathbf{P} \equiv \mathbf{D}=0$, which holds near the resonator surface, and from (13) we obtain at once the required relation (15). But it is better to obtain it also from the microscopic consideration. To this end, we note that the potential at a point on the metal surface is zero, being the sum of potentials from DL in helium and its image in a metal. If we move along the contour into helium, then the potential immediately after the helium $\mathrm{DL}$ is equal to the doubled potential of DL, because DL and its image give now contributions of the same sign. Further along the contour, the field strength from both DLs is zero (if we neglect the weak field from the ends of DL). Thus, the required potential difference is equal to the potential in helium formed by two DLs. Below, we will write the potential, keeping in mind the potential difference between the given point and the ground.

Consider a dipole $\mathbf{d}$ located in helium at the point $\mathbf{r}$. At the origin of coordinates, it creates the potential ${ }^{39}$

$$
\varphi=-\frac{\mathbf{d} \mathbf{r}}{\varepsilon r^{3}}
$$

We will determine the contribution of the first layer of helium to the potential difference between the electrode and the ground, by summing the contributions of all mi- croscopic dipoles composing DL and its image:

$$
\begin{aligned}
\varphi_{1} & =-\sum_{j} \frac{2 \mathbf{d}_{1} \mathbf{r}_{j}}{\varepsilon r_{j}^{3}}=-\frac{2}{\varepsilon \bar{R}_{1||}^{2}} \int d \phi \rho d \rho \frac{d_{1, z} \times z_{0}}{\left(z_{0}^{2}+\rho^{2}\right)^{3 / 2}}= \\
& =-\frac{4 \pi d_{1, z}}{\varepsilon \bar{R}_{1 \|}^{2}}
\end{aligned}
$$

where $\bar{R}_{1||}$ is the mean distance (along the surface) in the first layer, and the summation is carried out only over atoms of helium. The consideration of the mutual polarization of dipoles of first layers varies the result only by several percents. But the formulas become awkward, and we omit these corrections.

In the experiment $\underline{\underline{1}}$, a standing half-wave of second sound was created in the form

$$
T=T_{0}-0.5 \Delta T(z) \cos \left(\omega_{2} t\right), \Delta T(z)=\triangle T_{0} \cos \left(z \pi / L_{r}\right) .
$$

Here, $\omega_{2}$ is the frequency of second sound, and $z$ is reckoned from the resonator end in helium. Consider the electrode at the resonator end $(z=0)$. Oscillations of the helium temperature lead to oscillations of potential (15) due to the dependence of $\bar{R}_{1 \|}, \varepsilon$, and $d_{1, z}$ on $T$. We now expand $\varphi_{1}$ in a power series with respect to $T-T_{0}$, leaving the first nontrivial term:

$$
\begin{gathered}
\varphi_{1}(T)=\varphi_{1}\left(T_{0}\right)+\left(T-T_{0}\right) \partial \varphi_{1} / \partial T \\
\frac{\partial \varphi_{1}}{\partial T}=\frac{\partial \varphi_{1}}{\partial \bar{R}_{1 \|}} \frac{\partial \bar{R}_{1 \|}}{\partial T}+\frac{\partial \varphi_{1}}{\partial \varepsilon} \frac{\partial \varepsilon}{\partial T}+ \\
+\frac{\partial \varphi_{1}}{\partial d_{\mathrm{h}, z}} \frac{\partial d_{\mathrm{h}, z}}{\partial T}+\frac{\partial \varphi_{1}}{\partial d_{\mathrm{mir}, z}} \frac{\partial d_{\mathrm{mir}, z}}{\partial T} .
\end{gathered}
$$

Then we have

$$
\frac{\partial \varphi_{1}}{\partial \bar{R}_{1 \|}} \frac{\partial \bar{R}_{1 \|}}{\partial T}=-\frac{2}{3} \alpha_{1}^{s} \varphi_{1} .
$$

We denote $d_{\mathrm{h}, z} \equiv d_{\mathrm{h}}, d_{\mathrm{mir}, z} \equiv d_{\mathrm{mir}}$, and $d_{\mathrm{h}, z}+d_{\mathrm{mir}, z}=$ $d_{1, z} \equiv d_{1}$. The quantity $\alpha=-n^{-1} \partial n / \partial T$ is the thermal expansion coefficient. The indices $l$ and $s$ in $\alpha$ refer to liquid and solid states, respectively, and the numerical index counts the helium layer starting from the metal surface.

The quantity $d_{\mathrm{h}}$ (12) depends on $T$ via $\bar{R}_{1}$ and $\bar{R}_{2}$. Note that $\partial S_{7} / \partial T$ is negligible for liquid helium ${ }^{7}$. Therefore, $\frac{\partial d_{\mathrm{h}}}{\partial T} \approx \frac{\partial d_{\mathrm{h}}}{\partial R_{1}} \frac{\partial \bar{R}_{1}}{\partial T}+\frac{\partial d_{\mathrm{h}}}{\partial R_{2}} \frac{\partial \bar{R}_{2}}{\partial T}=-\frac{7 d_{\mathrm{h}}}{3}\left(\frac{\alpha_{1} \bar{R}_{1}}{R_{1}+R_{2}}+\frac{\alpha_{2} \bar{R}_{2}}{R_{1}+R_{2}}\right)$ and

$$
\frac{\partial \varphi_{1}}{\partial d_{\mathrm{h}}} \frac{\partial d_{\mathrm{h}}}{\partial T} \approx-\varphi_{1} \frac{7 d_{\mathrm{h}}}{3 d_{1}}\left(\frac{\alpha_{1}^{s} \bar{R}_{1}}{\bar{R}_{1}+\bar{R}_{2}}+\frac{\alpha_{2}^{l} \bar{R}_{2}}{\bar{R}_{1}+\bar{R}_{2}}\right) .
$$

The quantity $d_{\text {mir }}$ (11), (3) depends on $T$ via $z_{0}$ and $\omega_{\mathrm{pl}}$. We do not know exactly $\omega_{\mathrm{pl}}(T)$, but the thermal expansion coefficient $\alpha_{\mathrm{m}}$ for metals at helium temperatures is extremely small, by $5-6$ orders less than that for He $\mathrm{II}^{40}$. Since the derivative of $\omega_{\mathrm{pl}}=\sqrt{4 \pi e^{2} n_{\mathrm{e}} / m_{\mathrm{e}}}$ with respect 
to $T$ is proportional to $\alpha_{\mathrm{m}}$, it is small, and we omit it. Then $d_{\text {mir }}(T)=d_{\text {mir }}\left(z_{0}(T)\right)$ and

$$
\frac{\partial \varphi_{1}}{\partial d_{\mathrm{mir}}} \frac{\partial d_{\mathrm{mir}}}{\partial T} \approx-\varphi_{1} \alpha_{z} \frac{4 d_{\mathrm{mir}}}{3 d_{1}},
$$

where $\alpha_{z}=3 \frac{\partial z_{0}}{\partial T} \frac{1}{z_{0}}$. In (18), it remains to calculate $\frac{\partial \varphi}{\partial \varepsilon} \frac{\partial \varepsilon}{\partial T}$. Relation (94) from Appendix B yields

$$
\frac{\partial \varphi_{1}}{\partial \varepsilon} \frac{\partial \varepsilon}{\partial T} \approx \varphi_{1} \frac{\beta_{0} \delta_{0}}{T^{2}}+\varphi_{1} \alpha_{1}^{l}\left(1-\varepsilon^{-1}\right) .
$$

Near the surface metal, the DM of a helium atom consists of two parts: the stationary part $d_{1, z} \equiv d_{1}$ and the fluctuating one, with the mean modulus $\tilde{d}$. We note that relation (48) includes the total DM of an atom $d_{\text {in }}$, and, according to (48), $\delta_{0} \sim d_{\text {in }}^{2}$. Therefore, we need to replace $\delta_{0}$ in (22) by $\delta_{0} d_{\delta 1}^{2} / \tilde{d}^{2}$, where $d_{\delta 1}^{2} \simeq \tilde{d}^{2}+d_{1}^{2}$ is the square modulus of the total DM of a helium atom at the surface. As a result, we have

$$
\frac{\partial \varphi_{1}}{\partial \varepsilon} \frac{\partial \varepsilon}{\partial T}=\varphi_{1} \frac{\beta_{0} \delta_{0}}{T^{2}} \frac{d_{\delta 1}^{2}\left(p_{1}\right)}{\tilde{d}^{2}(\operatorname{svp})}+\varphi_{1} \alpha_{1}^{l}\left(1-\varepsilon^{-1}\right) .
$$

This formula is true, if the first layer of helium is liquid. If the first layer is solid, the value of $\frac{\partial \varphi_{1}}{\partial \varepsilon} \frac{\partial \varepsilon}{\partial T}$ will be somewhat changed, but of the order of that in (23). However, relation (23) gives a small contribution to $a$ (33), and we omit this correction in what follows. Finally, potential (17), (18) takes the form

$$
\begin{aligned}
\frac{\partial \varphi_{1}}{\partial T} & \approx-\frac{7 d_{\mathrm{h}}}{3 d_{1}}\left(\frac{\alpha_{1}^{s} \bar{R}_{1}}{\bar{R}_{1}+\bar{R}_{2}}+\frac{\alpha_{2}^{l} \bar{R}_{2}}{\bar{R}_{1}+\bar{R}_{2}}\right) \varphi_{1}- \\
& -\frac{2}{3} \alpha_{1}^{s} \varphi_{1}-\frac{4 d_{\mathrm{mir}}}{3 d_{1}} \alpha_{z} \varphi_{1} .
\end{aligned}
$$

The quantity $\alpha_{z}=3 z_{0}^{-1} \partial z_{0} / \partial T$ is determined by the distance $z_{0}$ from the helium atoms to the metal. The value of $\alpha_{z}$ can be estimated with the help of the relation $\alpha_{z} \sim \alpha D_{\mathrm{h}} / D_{\mathrm{m}}$, where $D_{\mathrm{h}} \approx 11 \mathrm{~K}$ is the depth of the interaction potential between two ${ }^{4} \mathrm{He}$ atoms, and $D_{\mathrm{m}}$ is the depth of the ${ }^{4} \mathrm{He}$ atom-metal potential. The estimate follows from the relation $\delta \bar{R} \sim \delta V \sim D$, where $V$ is the corresponding potential. In the case where the first layer of helium is solid, we have $\alpha_{z} \sim \alpha_{1}^{s} D_{\mathrm{h}} / D_{\mathrm{m}}$, and

$$
\begin{aligned}
\frac{\partial \varphi_{1}}{\partial T} & \simeq-\alpha_{2}^{l} \varphi_{1} \frac{7 d_{\mathrm{h}}}{3 d_{1}} \frac{\bar{R}_{2}}{\bar{R}_{1}+\bar{R}_{2}}- \\
& -\alpha_{1}^{s} \varphi_{1}\left(\frac{2}{3}+\frac{4 d_{\mathrm{mir}}}{3 d_{1}} \frac{D_{\mathrm{h}}}{D_{\mathrm{m}}}+\frac{7 d_{\mathrm{h}}}{3 d_{1}} \frac{\bar{R}_{1}}{\bar{R}_{1}+\bar{R}_{2}}\right) .
\end{aligned}
$$

In the presence of the wave of second sound (16), the potential $\varphi_{1}(T)$ oscillates with the frequency of second sound and with the amplitude

$$
\triangle \varphi_{1}(z)=\frac{\partial \varphi_{1}}{\partial T}\left(T_{0}\right) \Delta T(z) .
$$

It is convenient to represent $\Delta \varphi_{1}$ in the form

$$
\triangle \varphi_{1}(z)=\frac{k_{B} \Delta T(z)}{2|e|} a_{1},
$$

$$
\begin{gathered}
a_{1} \approx \frac{5.78 K n_{1}^{2 / 3}}{\varepsilon 10^{-4}|e| a_{B} n_{0}^{2 / 3}}\left\{\frac{7 \alpha_{2}^{l} d_{\mathrm{h}}}{1+\bar{R}_{1} / \bar{R}_{2}}+\right. \\
\left.+\alpha_{1}^{s}\left(2 d_{1}+d_{\text {mir }} \frac{4 D_{\mathrm{h}}}{D_{\mathrm{m}}}+\frac{7 d_{\mathrm{h}}}{1+\bar{R}_{2} / \bar{R}_{1}}\right)\right\} .
\end{gathered}
$$

Now let us consider the second and subsequent layers of helium. In helium near the wall, the pressure $p$ at the very wall is maximum (25 atm), but it drops to the bulk pressure as the distance from the wall increases by 2-3 atomic layers ${ }^{31}$. Due to the difference in pressures, the concentration of helium atoms in the first and third layers are different. Therefore, the tidal actions of these layers on the second layer are also different. As a result, the second layer is polarized. The third layer becomes polarized as well. But the polarization of the fourth layer is negligible, since the pressure in the third layer coincides almost with the bulk one $\underline{31}$. According to formula (20) in Ref. 7, the polarization of the $j^{\text {th }}$ layer is given by

$$
\begin{aligned}
\mathbf{d}_{j \geq 2} & \approx d_{0} \mathbf{i}_{z} \frac{n_{j-1} S_{7}\left(n_{j-1}\right)-n_{j+1} S_{7}\left(n_{j+1}\right)}{4 n_{0}}= \\
& =d_{0} \mathbf{i}_{z} \frac{S_{7}\left(n_{0}\right)}{4}\left\{\left(\frac{n_{j-1}}{n_{0}}\right)^{7 / 3}-\left(\frac{n_{j+1}}{n_{0}}\right)^{7 / 3}\right\} .
\end{aligned}
$$

(we note a small mistake in Ref. 7: the sign of $d_{0}$ in formula (11) is wrong; after its correction, the signs in the subsequent formulas become correct, and $d_{0}$ will have the sign in accordance with formula (9) of the present work). The polarization (11) induced by the metal is negligible for these layers. Similarly to the calculation in the case of the first layer, we obtain

$$
\begin{gathered}
\varphi_{j \geq 2}=-\frac{4 \pi d_{j}}{\varepsilon \bar{R}_{j \|}^{2}}, \\
a_{j \geq 2} \approx \frac{53.63 K \cdot n_{j}^{2 / 3}}{\varepsilon n_{0}^{3}}\left\{n_{j-1}^{7 / 3}\left(\frac{2}{7} \alpha_{j}+\alpha_{j-1}\right)-\right. \\
\left.-n_{j+1}^{7 / 3}\left(\frac{2}{7} \alpha_{j}+\alpha_{j+1}\right)\right\},
\end{gathered}
$$

where $\alpha_{j} \equiv \alpha_{j}\left(T_{j}, p_{j}\right)$. By considering all layers, we get

$$
\begin{gathered}
\varphi=\sum_{j=1}^{\infty} \varphi_{j}, \\
\Delta \varphi=\frac{k_{B} \triangle T}{2|e|} a, \quad a=\sum_{j=1}^{\infty} a_{j} .
\end{gathered}
$$

In our case, $a_{j \geq 4}$ are small and give a contribution to the bulk $\Delta \varphi$ calculated in Ref. 7. Therefore,

$$
a=a_{1}+a_{2}+a_{3} .
$$

We now determine the quantity $a$ for electrodes made of $\mathrm{Au}$ and $\mathrm{Cu}$, located at the resonator end $(z=0)$. 
TABLE I: The parameters of metals and the dipole moments $\left(d_{j}\right)$ of helium atoms in three first layers at the metal surface; the notation is given in the text. The values of $d_{\mathrm{h}}$ and $d_{j}$ for $\mathrm{Au}$ and $\mathrm{Cu}$ are given for $p_{2}=13 \mathrm{~atm}$.

\begin{tabular}{|c|c|c|c|c|}
\hline & $\mathrm{Au}$ & $\mathrm{Cu}$ & $\mathrm{Cs}$ & $\mathrm{Na}$ \\
\hline$z_{0}, \AA$ & 3.17 & 3.59 & 5.7 & 5.31 \\
\hline$D_{\mathrm{m}}, \mathrm{K}$ & 92.8 & 59.0 & 7.0 & 12.5 \\
\hline$\hbar \omega_{\mathrm{pl}}, \mathrm{eV}$ & 25.8 & 20 & 3.3 & 5.78 \\
\hline$\frac{d_{\operatorname{mir}}}{10^{-4}|e| a_{B}}$ & 2.92 & 1.52 & 0.056 & 0.12 \\
\hline$\frac{d_{\mathrm{h}}}{10^{-4}|e| a_{B}}$ & -2.06 & -2.06 & -1.32 & -1.32 \\
\hline$\frac{d_{1}}{10^{-4}|e| a_{B}}$ & 0.85 & -0.54 & -1.26 & -1.2 \\
\hline$\frac{d_{2}}{10^{-4}|e| a_{B}}$ & 1.16 & 1.16 & $\approx 0$ & $\approx 0$ \\
\hline$\frac{d_{3}}{10^{-4}|e| a_{B}}$ & 0.41 & 0.41 & 0 & 0 \\
\hline
\end{tabular}

In Table I, we present the known parameters of metals $\hbar \omega_{\mathrm{pl}}{ }^{20,41}, D_{\mathrm{m}}$, and $z_{0} \frac{26,42}{2}$, and those determined in the present work: $d_{\text {mir }}, d_{1}, d_{2}$, and $d_{3}$. If the temperature $T$ in all layers coincides with the bulk temperature of He II, the value of $a$ is an order of magnitude smaller than the experimental one, has opposite sign (the sign of $a$ determines the signal polarity), and increases with $T$ (see Fig. 1).

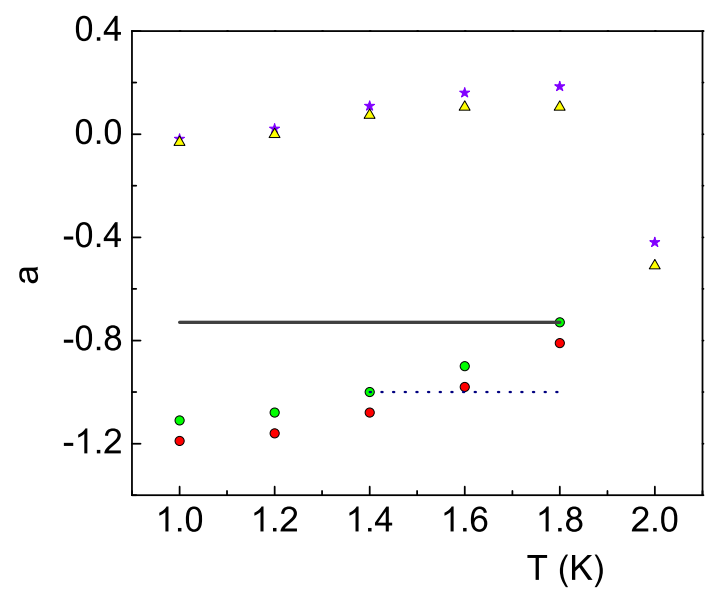

FIG. 1: Theoretical values of $a(T)$ determining the potential difference $\triangle U=a k_{B} \triangle T / 2|e|$ between an electrode at the resonator end and the ground: (a) without taking into account the influence of the metal on the temperature of the near-surface layers of helium: $\star$ denotes the quantity $a(T)$ for $\mathrm{Au}$, and $\triangle$ for $\mathrm{Cu}$; (b) taking into account the influence of the metal on the temperature of the first three near-surface layers of helium: o corresponds to $\mathrm{Au}$, and $\bullet$ to $\mathrm{Cu}$; (c) taking into account the influence of the metal on the temperature of the first four near-surface layers of helium: the solid line shows $a(T)$ for $\mathrm{Au}$. The dashed line is the experimental dependence $a(T)$ for $\mathrm{Au}$ and brass.
The experiment shows that $\rho_{s}=0$ at the wall. Since helium wets the wall, but $\rho_{s}$ increases to the bulk value only at a distance of $2-3$ atomic layers ${ }^{31}$ from the wall, the exhaustion of $\rho_{s}$ must be related not to the smallness of the total density, but to the behavior of quasiparticles at the wall (see Sec. III and, in more detail, Ref. 19). The equality of $\rho_{s}$ to zero is the condition for the $\lambda$-transition. Therefore, $T=T_{\lambda}$ at the wall, and $T$ decreases smoothly to the bulk value as the distance to the wall increases. Note that the distance between rotons at $T \simeq T_{\lambda}$ is about two interatomic distances, i.e., it is approximately equal to the roton size $(\sim 3$ atomic layers, according to the experimental scattering cross-section of rotons by vortex lines ${ }^{32}$ ). Hence, the free path of a roton does not exceed the interatomic distance. Therefore, we may consider the temperatures of separate atomic layers. If the temperature of helium is considerably smaller than $T_{\lambda}$, then the temperature of the first layers is determined by surface quasiparticles. As the bulk temperature is changed by $\delta T$, the temperature of the $j^{\text {th }}$ layer is changed by $\delta T_{j} \approx \delta T$. In the estimates, we use a linear law $T_{j}=T_{\lambda}\left(p_{j}\right)-j \cdot 0.1 \mathrm{~K}$. If the pressure and the temperature are equal to the bulk values, by starting from the $3^{\text {rd }}$ and $4^{\text {th }}$ (the $3^{\text {rd }}$ liquid one) layers, respectively, we obtain $p_{1} \gtrsim 30$ atm, $p_{2} \approx 13$ atm, $p_{j \geq 3}=\mathrm{svp}$, $n_{1} / n_{0} \approx 1.31, n_{2} / n_{0} \approx 1.123, n_{j>2} / n_{0}=1, T_{1} \approx 1.8 \mathrm{~K}$, $T_{2} \approx T_{3} \approx 2 \mathrm{~K}, T_{j \geq 4}=T$. We note that $T_{\lambda}$ depends on $p$. For $\alpha_{1} \equiv \alpha^{s}$, we used the data for the hcp phase $\mathrm{e}^{38}$ (solid helium can be present also in the bcc phase, but the interval of relevant pressures is very narrow). The resulting $a$ (see Fig. 1) for $\mathrm{Au}$ and $\mathrm{Cu}$ are close to the experimental values $a_{\exp } \approx-1$ and increase with the temperature. The experiment indicates that $a$ does not depend on $T$. Theoretically, this is obtained in the case where the temperature of the $4^{\text {th }}$ layer is also determined by the wall $(\approx 1.8 \mathrm{~K})$. Then $a_{\mathrm{Au}} \approx-0.73$ (the solid line in Fig. 1) and $a_{\mathrm{Cu}} \approx-0.81$.

Thus, with regard for the exhaustion of $\rho_{s}$, the theoretical value of $a$ corresponds approximately to the experimental one and does not depend on the temperature.

We note that, in the mode where the temperature of the first four layers is determined by the wall, we have $a_{1} \approx 3.05, a_{2} \approx 0.25, a_{3} \approx-4.03$, and $a=a_{1}+a_{2}+a_{3} \approx$ -0.73 for Au. As we can see, the first and third layers give the main contribution, but with different signs. This determines the sensitivity of $a$ to the value of $p_{2}$. The analysis indicates that, at $p_{2}=10 \mathrm{~atm}$, we obtain $a \approx 0.05$ for Au. For smaller values of $p_{2}$, the quantity $a$ increases and reaches $\sim 1$, which corresponds to the experimental value in magnitude but has opposite polarity. At $p_{2} \gtrsim 12$ atm, we have $a \sim-1$, i.e., the signal agrees with the experimental one in magnitude and polarity.

We do not consider the case where two or more solid atomic layers of helium are present near the metal, since no such metals are reliably known. 


\section{B. Electrodes with a low potential}

(i) Let helium wet a metal without solidifying at its surface. This is true for alkaline metals $\mathrm{Rb}, \mathrm{Na}, \mathrm{K}$, and Li. The analysis is similar to the group of Sec. IVA. The difference consists in that the first layer of helium is liquid, rather than solid. For these metals, the helium atom-metal potential is close to the ${ }^{4} \mathrm{He}-{ }^{4} \mathrm{He}$ potential. Hence, the metal does not attract strongly helium atoms, and the pressure in the first layers of helium near the metal is close to the bulk one (svp). Therefore, only the first layer of helium is polarized. For the rest of layers, we have $\nabla n=0$, and polarization is absent, according to (29). Relation (28) with $\bar{R}_{2}=\bar{R}_{1}=\bar{R}_{0}$ and $\alpha_{1}^{s} \rightarrow \alpha_{1}^{l}$, yields

$$
\begin{aligned}
a_{1} & \approx \frac{5.78 K}{\varepsilon 10^{-4}|e| a_{B}}\left\{\frac{7}{2} \alpha_{2}^{l} d_{\mathrm{h}}+\right. \\
& \left.+\alpha_{1}^{l}\left(2 d_{1}+d_{\operatorname{mir}} \frac{4 D_{\mathrm{h}}}{D_{\mathrm{m}}}+\frac{7}{2} d_{\mathrm{h}}\right)\right\}
\end{aligned}
$$

with $\mathbf{d}_{\mathrm{h}}$ given by (11). The resulting $a$ for Na are presented in Fig. 2. We have considered two cases: (1) $T_{1}$ and $T_{2}$ are equal to the bulk temperature, (2) $T_{1}$ and $T_{2}$ are determined by the wall $\left(T_{1} \approx T_{\lambda}(\operatorname{svp})-0.1 \mathrm{~K} \approx\right.$ $\left.2.07 \mathrm{~K}, T_{2} \approx T_{1}-0.1 \mathrm{~K}\right)$. In the first case, $a<1$ and rapidly increases with the temperature; in the second case, we have $a \approx 0.9$. The value of $a$ for $\mathrm{K}, \mathrm{Li}$, and $\mathrm{Rb}$ is almost the same as that for $\mathrm{Na}$ since the difference between these values is connected with $d_{\text {mir }}$, which is very small for these metals.

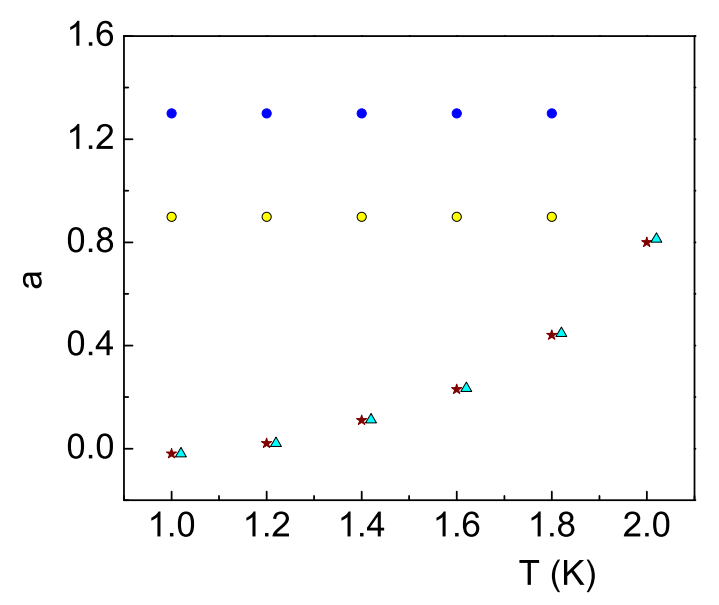

FIG. 2: The same as in Fig. 1. (a) Without taking into account the influence of a metal on the temperature of the near-surface layers of helium: the quantity $a(T)$ is given for $\mathrm{Na}(\star)$ and $\mathrm{Cs}(\triangle)$; (b) taking into account this influence: o corresponds to $\mathrm{Na}$, and $\bullet$ to $\mathrm{Cs}$.

(ii) Consider a metal that is not wetted by helium. Such a metal ${ }^{43}$ is Cs. Due to the nonwettability, the He II surface is separated from the metal surface by the distance $z_{0}$. To our knowledge, the question about the vanishing of $\rho_{s}$ at the free surface of He II remains to be open. The surface rotons are not "glued" to a metal, but they propagate along the free surface of He II. However, the dispersion curve of surface rotons should be close to that for the case where He II wets the metal. Therefore, the reasoning of Sec. III and Ref. 19 implies that $\rho_{s}$ must be exhausted at the free surface: $\rho_{s}=0$ and $T=T_{\lambda}$.

The quantities $\mathbf{d}_{\mathrm{h}}$ and $a$ are determined by formulas (11) and (35). We calculated $a$ for the same two cases as in item (i). In the case where $T_{1}$ and $T_{2}$ are determined by the wall, we need to take into account that $T_{\lambda}$ is attained at the surface of helium. Due to zero oscillations, the atoms are located not on the surface, but at a distance $\sim 1 \AA$, on the average, below it. Respectively, we have $T_{1} \approx T_{\lambda}(\mathrm{svp})-0.1 \mathrm{~K} \AA / \bar{R}_{0} \approx 2.14 \mathrm{~K}, T_{2} \approx T_{1}-0.1 \mathrm{~K} \approx$ $2.04 \mathrm{~K}$. The resulting $a$ is presented in Fig. 2 As one can see, the points coincide practically with those for $\mathrm{Na}$ in case (1). In case (2), we have $a \approx 1.3$, which is by a factor of 1.4 greater than the same quantity for $\mathrm{Na}$ and other alkaline metals. The possibility for Cs to possess a special value of $a$ has been already discussed in Ref. 8 .

The polarity $S$ of a signal is determined by the signs of $\varphi$ and $\partial \varphi / \partial T$. Let us denote the experimental $S$ by $S=(+)$. Then, according to the model, all metals of group A, i.e., $\mathrm{Au}$ and $\mathrm{Cu}$, have $S=(+)$, whereas the alkaline metals (Na, Cs, and others) have $S=(-)$.

\section{THE BULK POLARIZATION OF HELIUM INDUCED BY THE SURFACE DIPOLE LAYER.}

In work ${ }^{7}$, formula (37) presents the bulk polarization of helium arisen in a wave of second sound due to the gradients of the density (main contribution) and the temperature:

$$
\mathbf{P}(Z)=n \mathbf{d}(Z) / \varepsilon \approx 3.5 S_{7} d_{0} n \bar{R} \nabla T(Z) \alpha / 3 \varepsilon .
$$

In Ref. 7, the polarization was determined without $\varepsilon$ in the denominator, and $\varepsilon$ was considered in the potential. But more accurate to consider $\varepsilon$ namely in $\mathbf{P}$, because $\mathbf{d}$ is the proper DM of an atom and is screened by the medium. Such a polarization induces the electric signal on the electrode that is weaker by one-two orders of magnitude than the surface signal determined in the previous section. However, there exists one more source of the bulk polarization that was not considered in Ref. 7 . It is the above-considered surface DL (dipole layer).

According to the analysis in the previous section, DL consisting of several strongly polarized layers of helium atoms is formed on all internal surfaces of the resonator. Values of DM of an atom in three first layers are given in Table 1. Let us consider two inner end surfaces of a cylindrical resonator. As known, the infinite DL creates no field $\mathbf{E}$ outside of itself. But the real layer is a thin disk with finite radius equal to the resonator radius $R_{r}$. Such a layer creates the field outside of itself as well. The 
first layer of DL creates the potential

$$
\begin{aligned}
\varphi_{1}(\mathbf{R}) & =\sum_{j} \frac{2 \mathbf{d}_{1}\left(\mathbf{R}-\mathbf{r}_{j}\right)}{\varepsilon\left|\mathbf{r}_{j}-\mathbf{R}\right|^{3}}=\frac{2 n_{2,1} d_{1} Z}{\varepsilon} \int_{0}^{2 \pi} d \phi \times \\
& \times \int_{0}^{R_{r}} \frac{\rho d \rho}{\left(Z^{2}+\rho^{2}+R^{2}-2 R \rho \cos \phi\right)^{3 / 2}}= \\
& =\frac{4 \pi n_{2,1} d_{1}}{\varepsilon}\left(1-\frac{Z f(Z, R)}{\sqrt{Z^{2}+R_{r}^{2}}}\right),
\end{aligned}
$$

in helium at a point $\mathbf{R}=(R, 0, Z)$. Here, $\mathbf{R}_{j}=(\rho, \phi, 0)$ are coordinates of the $j^{\text {th }}$ atom of helium in the layer, $n_{2, l}$ is the surface concentration of helium atoms in the $l^{\text {th }}$ layer, and

$$
\begin{aligned}
f(Z, R) & =\frac{\sqrt{\tilde{Z}^{2}+1}}{\pi} \int_{0}^{\pi} d \phi \frac{\left(\tilde{Z}^{2}+\tilde{\rho}^{2}+\tilde{\rho} \cos \phi\right)}{\left(\tilde{Z}^{2}+\tilde{\rho}^{2} \sin ^{2} \phi\right)} \times \\
& \times\left(\tilde{Z}^{2}+\tilde{\rho}^{2}+1+2 \tilde{\rho} \cos \phi\right)^{-1 / 2}
\end{aligned}
$$

where $\tilde{Z}=Z / R_{r}, \tilde{\rho}=R / R_{r}$. For the points on the resonator axis, we have $f=1$. In Fig. 3. we show the numerically determined dependence of the function $Z f(Z, R)\left(Z^{2}+R_{r}^{2}\right)^{-1 / 2}$ on $Z$ at several $R$, by comparing it with the same function at $f=1$. The exact factor $f$ flattens the dependence on $Z$ and weakens the strength $E_{z}$. We note that formulas (37) and (38) do not involve the contribution of images of the dipoles arising on the electrode and walls of the resonator. Below, we will use formula (38) with $f=1$ for estimates. Then the integrals can be calculated analytically.

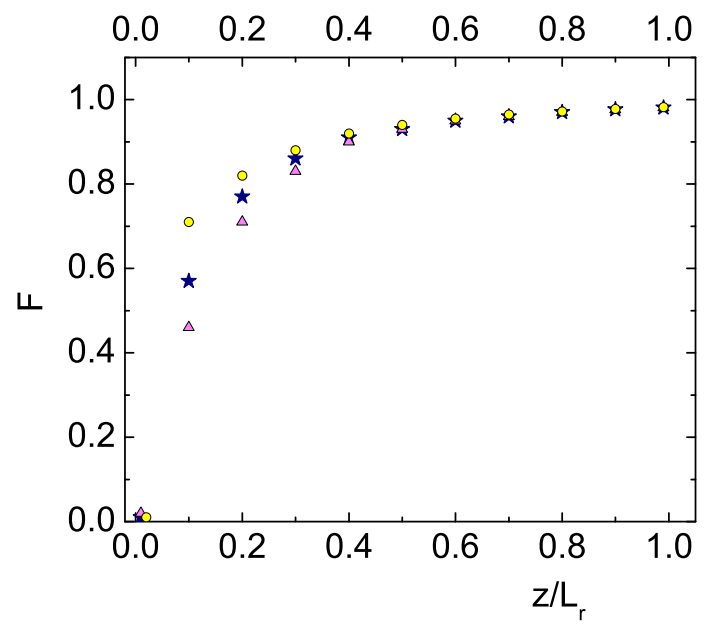

FIG. 3: Dependence of the function $F=z f(z)\left(z^{2}+R_{r}^{2}\right)^{-1 / 2}$ on $z$ at $\sigma=0.2$ and several values of $R: R \leq 0.2 R_{r}(\triangle), R=$ $0.7 R_{r}(\star)$, and $R=0.99 R_{r}(\bullet)$. For points on the resonator axis $(R=0)$, we have $f=1$; the corresponding $F(z)$ coincides with the curve $\triangle \triangle \triangle$. At $z \gtrsim 0.5 L_{r}$, three curves merge to the one curve.
Considering that DL is present on both ends of the resonator and consists of three atomic layers and taking $f=1$, we obtain the total potential:

$$
\begin{gathered}
\varphi(\mathbf{R}) \approx \frac{4 \pi n_{2, e f} d_{e f}}{\varepsilon} f_{2}(Z)=-\varphi_{s} f_{2}(Z), \\
f_{2}(Z)=\left(2-\frac{Z}{\sqrt{Z^{2}+R_{r}^{2}}}-\frac{L_{r}-Z}{\sqrt{\left(L_{r}-Z\right)^{2}+R_{r}^{2}}}\right) .
\end{gathered}
$$

Here, $\varphi_{s}$ is the surface potential on the electrode from three layers given by (33), and $n_{2, \text { ef }} d_{\text {ef }}=n_{2,1} d_{1}+$ $n_{2,2} d_{2}+n_{2,3} d_{3}$. Potential (39) creates the field $\mathbf{E}=$ $-\mathbf{i}_{z} \partial \varphi / \partial Z-\mathbf{i}_{\rho} \partial \varphi / \partial R$. If we consider the exact value of $f$, the analysis indicates that the $E_{\rho}$ component is of the order of magnitude of the $z$-component but is somewhat less. In the approximation where $f=1$, we have $E_{\rho}=0$, and the $z$-component, $E_{z}$, induces the bulk polarization

$$
\begin{aligned}
& \frac{n d_{z}}{\varepsilon}=P_{z}=\frac{(\varepsilon-1) E_{z}}{4 \pi}=\frac{(\varepsilon-1) \varphi_{s}}{4 \pi} \times \\
\times & \left(\frac{R_{r}^{2}}{\sqrt{Z^{2}+R_{r}^{2}}}-\frac{R_{r}^{2}}{\sqrt{\left(L_{r}-Z\right)^{2}+R_{r}^{2}}}\right)
\end{aligned}
$$

in helium. As was noted above, taking into account the exact function $f$ causes a decrease in both $E_{z}$ and the polarization. On the end electrode $(Z=0)$, polarization (41) induces the potential

$$
\begin{gathered}
\varphi_{b s} \approx-\sum_{j} \frac{\mathbf{d}\left(\mathbf{r}_{j}\right) \mathbf{r}_{j}}{\varepsilon r_{j}^{3}}=\frac{(\varepsilon-1) \varphi_{s} I_{b s}}{2}, \\
I_{b s}=\sigma^{2} \int_{0}^{1} d z\left(1-\frac{z}{\sqrt{z^{2}+\sigma^{2}}}\right) \times \\
\quad \times\left(\frac{1}{\left(z^{2}+\sigma^{2}\right)^{3 / 2}}-\frac{1}{\left((1-z)^{2}+\sigma^{2}\right)^{3 / 2}}\right),
\end{gathered}
$$

where $\sigma=R_{r} / L_{r} \lesssim 1$. However, one need to take into account that we are interested in the variable part of the potential. Therefore, one should change the sign of the contribution of the second resonator end (in Eqs. (41) and (43)), because the value of $\delta T$ has different sign at different resonator ends, in the half-wave of second sound. So, we obtain for $I_{b s}$ in Eq. (42):

$$
\begin{aligned}
I_{b s} & =\sigma^{2} \int_{0}^{1} d z\left(1-\frac{z}{\sqrt{z^{2}+\sigma^{2}}}\right) \times \\
& \times\left(\frac{1}{\left(z^{2}+\sigma^{2}\right)^{3 / 2}}+\frac{1}{\left((1-z)^{2}+\sigma^{2}\right)^{3 / 2}}\right) .
\end{aligned}
$$

The numerical calculation for (44) gives $I_{b s} \approx 0.38 ; 0.7$ at $\sigma=1 / 24 ; 1 / 2$ ( $\sigma$ in first experiment), $I_{b s} \approx 0.51$ at $\sigma=0.2$ (new experiment). As a result, the bulk potential $\varphi_{b s}$ is equal to the above-calculated surface potential 
$\varphi_{s}$ multiplied by a small factor $(\lesssim 0.02)$ that depends on the ratio of resonator sizes. Such a potential leads to a small correction to signal (33), in the limits $\sim 2 \%$. This correction is larger for short resonators, as for polarization (36).

Polarization (41) is much greater than polarization (36). But the main part of (41) is invariable in time and unobservable. The variable part is, on the average, by one order of magnitude less than polarization (36), their ratio $\sim-0.1 a \alpha(1.8 K) / \alpha(T)$. In this case, the strength of the field induced by DL turns out, on the average, approximately by one order of magnitude more than the strength induced by a wave of the spontaneous polarization (36).

Besides ends, DL is present also on the lateral surfaces of a resonator. We omit the calculation of the potential of this DL, since a lateral surface can be divided into segments and represent in the form of a fan of pairs of almost plane surfaces positioned oppositely to each other. By this, the problem is reduced to the previous one, and, hence, the signal should be of the same order, i.e., it should be low. The exact calculation without regard for the above approximations is complicated and can be performed only numerically. However, the above-found estimate must give the true order of magnitude.

Thus, DL on the internal surface of a resonator induces the polarization in the bulk of helium that is variable in time and approximately by one order of magnitude less than spontaneous polarization (36) related to the density gradient. The distribution of the induced polarization is such that the electric signal from it turns out low, $\sim 1 \%$ of the surface signal. Though, for a long resonator from the first experiment $\stackrel{1}{ }$, this signal is of order of magnitude of the signal from polarization (36) equal to ${ }^{7} \sim 0.1-1 \%$ of the surface signal. Thus, we will neglect the bulk signal (42), since it gives only a small correction to the surface signal.

Note that the potential (39) with opposite sign represents the more exact formula for the surface signal, as compared with (33), because it considers the contribution of the remote resonator end. Consideration of the DL on a lateral walls of the resonator will lead to an additional correction. All these corrections represent, in essence, a bulk ones. Due to them, the surface signal acquires a weak dependence on the resonator sizes, which is apparent from Eqs. (39) and (40). Here, we do not consider a possible difference between materials of the resonator ends. It is of interest that a weak dependence of the signal on the resonator sizes should be even at purely surface nature of the signal.

\section{INFLUENCE OF ADMIXTURES ON THE SIGNAL INDUCED BY SECOND SOUND.}

We now estimate the influence of admixtures on the polarization of helium and the amplitude of the electric signal. This question was not studied earlier.
First, we consider an admixture of nonpolar molecules or atoms. As an example, we consider the admixture of ${ }^{3} \mathrm{He}$ atoms, since their properties are studied quite well. The equations for the sounds for ${ }^{3} \mathrm{He}^{-}{ }^{4} \mathrm{He}$ mixtures are modified 44 . A change of the temperature of the mixture by $\triangle T$ leads to a change in the concentration of ${ }^{3}$ He atoms, according to equations (54.6) and (57.3) from Ref. 44:

$$
\begin{gathered}
\triangle f=\frac{f \Delta T \times \partial S_{\text {mix }} / \partial T+f \triangle p \times \partial S_{\text {mix }} / \partial p}{S_{\text {mix }}-f \times \partial S_{\text {mix }} / \partial f}, \\
S_{\text {mix }}=S_{0}+k_{B} n_{4} f \ln \left[\frac{2}{n_{4} f}\left(\frac{m^{*} k_{B} T}{2 \pi \hbar^{2}}\right)^{3 / 2}+\frac{5}{2}\right], \\
f=\frac{n_{3} m_{3}}{n_{3} m_{3}+n_{4} m_{4}},
\end{gathered}
$$

where $S_{\text {mix }}$ is the entropy of the mixture per unit volume, $S_{0}$ is the entropy of pure ${ }^{4} \mathrm{He}, n_{3}$ and $n_{4}$ are the concentrations of ${ }^{3} \mathrm{He}$ and ${ }^{4} \mathrm{He}$ atoms, respectively, and $m^{*} \approx m_{3}+m_{4}$ is the effective mass of the He atom in the mixture. For the second sound, we have

$$
\triangle f \approx f \triangle T \frac{1}{S_{0}} \frac{\partial S_{0}}{\partial T},
$$

hence, the full concentration, equal to $n_{\text {mix }}=n_{3}+n_{4} \approx$ $\left[1+4 f(3-3 f)^{-1}\right] n_{4}$, acquires an additional contribution from $\triangle f$ :

$$
\triangle n_{\text {mix }} \approx \frac{4 f}{3(1-f)^{2}} n_{4} \Delta T \frac{1}{S_{0}} \frac{\partial S_{0}}{\partial T} .
$$

At $T=1.4-2 \mathrm{~K}$ and $f=0.1$, we obtain $\Delta n_{\operatorname{mix}} \simeq$ $0.4 n_{\text {mix }} \Delta T / \mathrm{K}$, which is two orders of magnitude larger than the quantity $\triangle n_{4} \approx-n_{4} \alpha \triangle T$ for the second sound in pure ${ }^{4} \mathrm{He}$. If ${ }^{3} \mathrm{He}$ atoms do not form pairs with ${ }^{4} \mathrm{He}$ (the idea of such pairing is proposed in Ref. 45), then the polarizational properties of ${ }^{3} \mathrm{He}$ and ${ }^{4} \mathrm{He}$ atoms should be similar. In this case, an admixture of ${ }^{3} \mathrm{He}$ atoms will lead to an additional signal $U$, proportional to $f$. This signal consists of the (main) surface part and the volume part, as for the pure ${ }^{4} \mathrm{He}$. We can estimate the surface part by multiplying the signal (33) for the pure ${ }^{4} \mathrm{He}$ by the ratio $\Delta n_{\text {mix }}$ to $\Delta n_{4} \approx-n_{4} \alpha \Delta T$, all taken at the temperature of the first layer of the helium near the electrode $(T \simeq 2 \mathrm{~K})$. We obtain that, at high concentration of ${ }^{3} \mathrm{He}$ $(f=0.1)$, the additional signal in the wave of the second sound, for the golden electrode, must be one or two orders of magnitude larger than the already observed signal $\triangle U \approx-k_{B} \Delta T / 2|e|$. If ${ }^{3} \mathrm{He}$ atoms pair with ${ }^{4} \mathrm{He}$, then the effect should probably be stronger, since such molecules are electrically more active then the spherically symmetric ${ }^{3}$ He atoms. Thus, the random admixtures of nonpolar molecules do not affect the properties of a signal at low concentrations, but they can significantly enhance both surface and bulk signals at high concentrations. 
We now carry on several estimates for admixtures of polar molecules. Let the molecules be located in the bulk of He II. The electric field $\mathbf{E}$ induced by atoms of helium will put the proper DMs of molecules in order. So, the DMs will be so oriented to decrease the external field. Let us estimate this effect. The mean projection of the proper DM $\mathbf{d}_{p}$ of a molecule on the external field $\mathbf{E}$ is determined by the well-known formula

$$
d_{E}=\frac{d_{p}^{2} E}{3 k_{B} T} .
$$

In the bulk, the field $\mathbf{E}$ is different at different points and is directed mainly along the $Z$ axis of a resonator. A method of calculation of the electric field in a spontaneously polarized dielectric is discussed below in Appendix A. According to this method, the potential at a point with coordinates $\mathbf{R}=(R, 0, Z)$ can be determined as in Refs. 6.7, by summing the potentials from all local dipoles in the resonator:

$$
\begin{aligned}
& \varphi_{b}(\mathbf{R})=n \int d \mathbf{r} \frac{\mathbf{d}(z)(\mathbf{R}-\mathbf{r})}{\varepsilon|\mathbf{r}-\mathbf{R}|^{3}}= \\
& =\quad \int d V \frac{n d_{z}(z)(Z-z)}{\varepsilon\left[(z-Z)^{2}+R^{2}+\rho^{2}-2 R \rho \cos \phi\right]^{3 / 2}} .
\end{aligned}
$$

Here, $\mathbf{r}=(\rho, \phi, z)$ are the coordinates of helium atoms. We position the coordinate origin on the resonator axis at the center of the left end surface of the resonator. Using the DM (36) of helium atoms that arises due to the density gradient in a wave of second sound, we obtain

$$
\begin{gathered}
\varphi_{b}(\mathbf{R})=\varphi_{0} \gamma_{b}(Z, R, \sigma) \cos \left(\omega_{2} t\right), \\
\varphi_{0} \approx \frac{7 \pi S_{7} \alpha_{T} d_{0}}{12 \varepsilon \bar{R}^{2}} \Delta T_{0},
\end{gathered}
$$

where $\sigma=R_{r} / L_{r}$. We note that only the quantity

$$
\begin{gathered}
\gamma_{b}(Z, R, \sigma)=\sigma^{-1} \int_{0}^{2 \pi} d \phi \int_{0}^{1} d z \int_{0}^{1} d \rho \rho \sin (\pi z)(Z-z) \times \\
\times\left[(z-Z)^{2} / \sigma^{2}+(R-\rho)^{2}+2 R \rho(1-\cos \phi)\right]^{-3 / 2}
\end{gathered}
$$

depends on the observation point $Z, R$. In the formula (54), $R$ and $\rho$ are normalized to $R_{r}$, and $z, Z$ - to $L_{r}$. In the new experiment, the resonator length $L_{r}=2.5 \mathrm{~cm}$, and its radius $R_{r}=0.5 \mathrm{~cm}$. Therefore, $\sigma=0.2$. We determined the dependence $\gamma_{b}$ on $Z$ at $\sigma=0.2$ for several $R$ numerically by (54) and present the results in Fig. 4. For other $\sigma$, the solution of (54) can be found also numerically, and, approximately, the relation $\gamma_{b}(Z, R, \sigma) \simeq \gamma_{b}(Z, R, 0.2) \sigma / 0.2$ holds for all $\sigma \leq 1$. The electrode occupies approximately a half of the end surface of the resonator. So, the electrode is rather large, and we must average the potential over all points. But since the potential differs insignificantly at points with different $R$ (see Fig. (4), we can neglect this difference

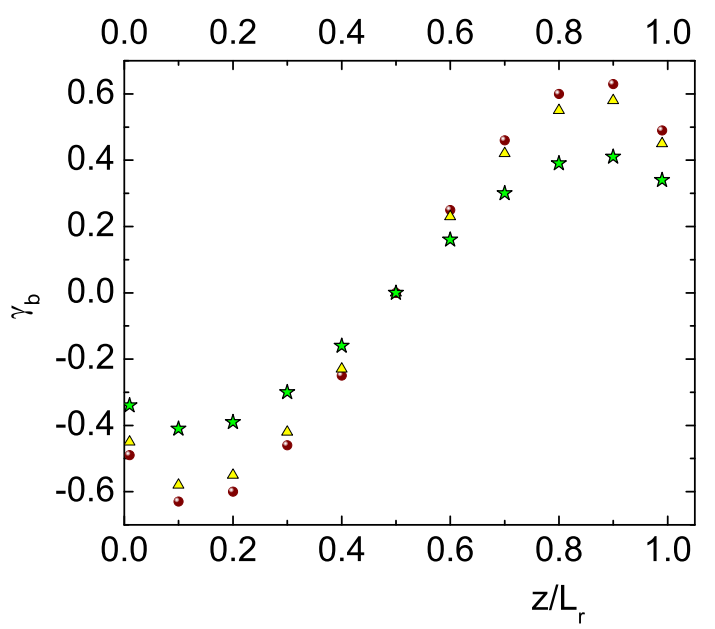

FIG. 4: Dependence of the quantity $\gamma_{b}$ (54) on $z$ at $\sigma=0.2$ and $R=0(\bullet), R=R_{r} / 2(\triangle), R=R_{r}(\star)$.

and consider the potential equal to that at the point at the electrode center $(R=0, Z \rightarrow 0)$.

The field strength

$\mathbf{E}=-\mathbf{i}_{z} \frac{\partial \varphi_{b}}{\partial Z}-\mathbf{i}_{\rho} \frac{\partial \varphi_{b}}{\partial R}=-\varphi_{0} \cos \left(\omega_{2} t\right)\left(\mathbf{i}_{z} \frac{\partial \gamma_{b}}{\partial Z}+\mathbf{i}_{\rho} \frac{\partial \gamma_{b}}{\partial R}\right)$

can be also determined numerically. With good accuracy, we have $\mathbf{E} \approx \mathbf{i}_{z} E_{z}, E_{z}=-P_{z}\left(\partial \gamma_{b} / \partial Z\right) \sin ^{-1}\left(\pi Z / L_{r}\right)$. For example, under the experimental conditions with $T=1.8 \mathrm{~K}$ and $\sigma=0.2$, we have $E_{z} \approx$ $0.1 k_{B} \triangle T_{0} \cos \left(\omega_{2} t\right) /|e| L_{r} \approx-2.5 P_{z}$ at the resonator cen$\operatorname{ter}\left(R=0, Z=0.5 L_{r}\right)$. At the beginning of the resonator axis $(R=0, Z \rightarrow 0), E_{z} \approx 0.06 k_{B} \triangle T_{0} \cos \left(\omega_{2} t\right) /|e| L_{r} \approx$ $-1.4 P_{z} / \sin \left(\pi Z / L_{r}\right) \approx-1.4 P_{z} L_{r} / \pi Z$, i.e., the polarization is much less than the field strength, since $L_{r} / Z \gg 1$. In this case, it is necessary to take also into account the induced polarization $\mathbf{P}=\kappa \mathbf{E}$ that determines the values of $\mathbf{P}$ at $Z \rightarrow 0, L_{r}$. The numerical analysis indicates that the consideration of the induced polarization leads to a slight change of potential (52): approximately by $1 \%$. At the points near the resonator center, the change is greater, but the potential is low there. Hence, such a correction can be neglected. We note that the consideration of the "images" of dipoles (arising in the electrode and walls of the resonator) in (54) should change potential (52) significantly, by several times (formula (54) involves no images).

The proper DMs of polar molecules can be very different. As usual $\frac{46}{6}$, the two-atom molecules have $d_{p} \simeq$ $0.5|e| a_{B}$, though DMs of some molecules are much less $\underline{46}$ (e.g., for molecules $\mathrm{CO}$ and $\left.\mathrm{NO}, d_{p} \simeq 0.05|e| a_{B}\right)$. In field (55), the molecules are ordered and acquire a directed $\mathrm{DM}$ (50). At $d_{p} \simeq 0.5|e| a_{B}$ under the experimental conditions with $T=1.8 \mathrm{~K}, \Delta T_{0} \simeq 1 m K$, we obtain that the directed DM is equal to $d_{E} \simeq 10^{-4}|e| a_{B}$, which exceeds a tidal DM $d_{0}$ (9) by a factor of $\simeq 3$. In this case, the 
bulk polarization (36) of helium atoms at $T=1.8 \mathrm{~K}$ is $d_{z} \simeq 2.7 \times 10^{-12} d_{0}$. This allows us to draw a significant conclusion that an admixture of polar molecules compensates the bulk polarization of helium, if the share of an admixture (the ratio of the concentration of an admixture to the concentration of helium) exceeds $\sim 10^{-12}$ ! It is a very small number: for example, the share of surface atoms of helium $\left(\sim 10^{-8}\right)$ is larger by 4 orders of magnitude. Most probably, helium contains always an admixture of polar molecules with a share of $\sim 10^{-12}$. In this case, the bulk polarization is absent always in second-sound waves.

In Sec. $\mathrm{V}$, one more kind of the bulk polarization of helium that is due to the dipole layer covering the internal surface of a resonator is found. This polarization is less by one order of magnitude than that by (36), but the field strength for it by one order of magnitude higher than that by (55). Therefore, such a polarization can be completely compensated by an admixture with a share of $\sim 10^{-14}$. In what follows, this polarization will be omitted.

The case of the surface polarization related to polar molecules is more complicated. If a molecule is very close to the surface, then it is attracted by the van der Waals forces, like nonpolar atoms. Moreover, since the molecule has proper DM, the mirror image of this DM appears in the medium adjacent to helium (the image is exact if the medium is a metal and is weakened if the medium is a dielectric), and we have an additional strong attraction of the dipole-dipole type. The orientation perpendicular to the surface is favorable for a dipole. In this case, the interaction energy

$$
W=\frac{\mathbf{d}_{1} \mathbf{d}_{2}-3\left(\mathbf{d}_{1} \mathbf{n}_{12}\right)\left(\mathbf{d}_{2} \mathbf{n}_{12}\right)}{\varepsilon R_{12}^{3}}=\frac{-2 d_{1}^{2}}{\varepsilon R_{12}^{3}},
$$

where $\mathbf{n}_{12}=\mathbf{R}_{12} / R_{12}, \mathbf{R}_{12}$ is the radius-vector from the dipole $\mathbf{d}_{1}$ to its image $\mathbf{d}_{2}$. Here, we take into account that $\mathbf{d}_{2}=\mathbf{d}_{1}$ for a metal. Let $d_{1}=d_{p} \simeq 0.5|e| a_{B}$. If there is no solid layer of helium on the surface, we have $R_{12} \simeq 2 \bar{R} \approx 7.2 \AA$ and $W \approx-60.5 \mathrm{~K}$. Such binding energy is much more than the energy of any quasiparticle in helium, so that practically all atoms of an admixture should be condensed on the surface. However, for molecules with a small DM $d_{p} \simeq 0.05 e a_{B}$, we obtain $W \approx-0.6 \mathrm{~K}$. At such small binding energy, quasiparticles will separate easily a molecule from the surface. A molecule can be held near the surface only by the van der Waals forces, like atoms of helium themselves. Since the surface attracts atoms of helium weakly, we may expect that it attracts atoms of the admixture in the same manner. Such an admixture should be floating freely in the bulk and should be able to damp the bulk polarization of helium. However, due to a small $d_{p}$, the critical share of an admixture is greater by two orders of magnitude: $\sim 10^{-10}$. If the surface attracts atoms of helium strongly, and one solid layer of atoms of helium is formed on the surface, then $R_{12} \simeq 4 \bar{R} \approx 14.4 \AA$. For $d_{p} \simeq 0.5 e a_{B}$, we have in this case $W \approx-7.6 \mathrm{~K}$. Such binding energy is easily overcome by a single roton. If the van der Waals interaction is taken into account, the value of $W$ increases by several times (in modulus). But we can expect that the total $|W|$ is less than the energy of three-four rotons. In the case where $T>1 \mathrm{~K}$ and the number of rotons is great (and near the surface their concentration, apparently, is maximum ${ }^{19}$ ), the number of molecules of an admixture in the bulk will exceed that on the surface. The exact proportion can be calculated. Such admixtures are also able to damp the bulk polarization. Some admixtures can form chemical bonds with a surface, but we will not consider this case.

Since the dipoles of surface molecules of an admixture are oriented, on the average, perpendicularly to the surface, they create a double charged layer and induce a potential on the electrode. We now estimate it in the case where an admixture covers all the surface by a single atomic layer. The potential created by an admixture on the electrode is determined by formula (15), where the quantities $\bar{R}_{1||}^{-2}$ and $d_{1, z}$ must be replaced, respectively, by the surface concentration of an admixture and by the $z$-component of DM of a molecule. In order to determine the variable signal from an admixture, we need to know the derivative $\partial \varphi / \partial T$. By differentiating, we obtain

$$
\frac{\partial \varphi}{\partial T} \simeq-\frac{2 \varphi \alpha_{a d}}{3}+\frac{\varphi}{d_{1, z}} \frac{\partial d_{1, z}}{\partial T} .
$$

Let us assume that the coefficient of thermal expansion $\alpha_{a d}$ of an admixture is about $\alpha$ of liquid helium. Then, at $d_{1, z} \simeq d_{p} \simeq 0.5|e| a_{B}$, only the first term in (57) is related to the appearance of an electric signal that is by 4 orders of magnitude greater than the surface signal (33) from atoms of helium. We do not know how the second term in (57) can be simply estimated. But we can expect that the projection of a dipole reacts to a change of the temperature stronger than the concentration, therefore the contribution of this term should be significantly greater than that from the first term in (57). Thus, a single filled layer of an admixture of polar atoms gives the surface signal up to $10 \mathrm{mV}$, that is greater by $4-5$ orders of magnitude than the signal from atoms of helium. If atoms of an admixture fill the layer only partially or, conversely, there are many layers, the signal will change proportionally.

The estimates are very approximate for the surface polarization from polar atoms and are more exact for the bulk polarization. For the signal from the admixture of ${ }^{3} \mathrm{He}$ atoms, the estimates are quite reliable. In two last cases, the order of magnitude must be correct. It is seen from these estimates that admixtures can strongly affect the signal, by increasing or decreasing it. As sufficiently unexpected, we mention the result on a possible complete damping of the bulk polarization of helium by an admixture of polar molecules. Qualitatively, this damping is related to the extreme smallness of the bulk polarization of helium: it is less by $\sim 12$ orders of magnitude than the surface polarization and by $\sim 16$ than the DM $d_{p}$ of polar molecules. Therefore, the latter can easily compen- 
sate the bulk polarization. To clarify the role of admixtures, a more detailed analysis should be performed. The principal question is whether the critical concentration of bulk polar admixtures is reached.

We note that the experimental signal can be caused, in principle, by a layer of polar molecules on the electrode with the concentration $\sim 10^{-4}$ of that of helium. This is seen from the above estimates. But then the signals must be different in different experiments, which was not observed. Therefore, the signal is related namely to atoms of helium, and the theory of such a signal (see above) is in good agreement with experiments.

It would be of interest to experimentally investigate the role of admixtures.

\section{THE ELECTRIC SIGNAL INDUCED BY A FIRST-SOUND WAVE}

According to the model, the signal in a wave of second sound arises for two reasons. Due to the isotropy breaking in the system, the helium atoms are strongly polarized in the region near the electrode, and the polarization oscillates due to oscillations of the density of helium. In fact, the signal is determined by the low-intensity first sound accompanying second sound. Then the electric signal undoubtedly should be observed after excitation of a standing half-wave of the first (as the main) sound in helium. However, such a signal was not observed in the experiment ${ }^{1}$. Below, we will calculate the expected magnitude of the signal.

In a standing half-wave of first sound in the harmonic mode, the pressure varies according to the law

$$
p=p_{0}-0.5 \triangle p(z) \cos \left(\omega_{1} t\right), \triangle p(z)=\triangle p_{0} \cos \left(z \pi / L_{r}\right)
$$

(and similarly for the density); $\triangle p(z)$ and $\triangle \rho(z) \equiv$ $\triangle \rho_{0} \cos \left(z \pi / L_{r}\right)$ are the amplitudes of oscillations of the pressure and density of helium, respectively, at points with coordinate $z$.

The signal observed in a wave of second sound can be written in the form (33) just from the dimensional analysis. The experiment gives $a \approx-1$. The quantity $a$ must be calculated in theory. Starting from the dimensional consideration, we have, for first sound,

$$
\frac{\triangle \varphi(z)}{\triangle p(z)}=\frac{b}{|e| n} \approx b \cdot 2.89 \cdot 10^{-5} \frac{\mathrm{V}}{\mathrm{atm}},
$$

where $n$ is the concentration of He II. Since the nearsurface polarization arises on atomic scales, we may expect that $|b| \sim 1$.

Similarly to second sound, first sound induces surface and bulk signals. We will determine the surface signal analogously to the case of second sound, by expanding the potential in the density (instead of temperature) and assuming that $\triangle p$ is the same in different near-surface layers. In this way, we obtain:

$$
b_{2 D}=b_{1}+b_{2}+b_{3}+\ldots,
$$

$$
\begin{aligned}
& b_{1} \approx \frac{|e| \varphi_{1} \rho_{0}}{3 m_{4} \rho_{1} u_{1}^{2}\left(p_{1}\right)}\left\{2+7 \frac{d_{\mathrm{h}}}{d_{1}}\left(\frac{1}{1+\bar{R}_{2} / \bar{R}_{1}}+\right.\right. \\
& \left.\left.+\frac{\rho_{1} u_{1}^{2}\left(p_{1}\right)}{\rho_{2} u_{1}^{2}\left(p_{2}\right)} \frac{1}{1+\bar{R}_{1} / \bar{R}_{2}}\right)\right\} \text {, } \\
& b_{j \geq 2} \approx \frac{|e| \varphi_{j}}{3 m_{4} u_{1}^{2}\left(p_{j}\right)}\left\{\frac{2 \rho_{0}}{\rho_{j}}+\right. \\
& \left.+7 \frac{\frac{u_{1}^{2}\left(p_{j}\right)}{u_{1}^{2}\left(p_{j-1}\right)}\left(\frac{\rho_{j-1}}{\rho_{0}}\right)^{4 / 3}-\frac{u_{1}^{2}\left(p_{j}\right)}{u_{1}^{2}\left(p_{j+1}\right)}\left(\frac{\rho_{j+1}}{\rho_{0}}\right)^{4 / 3}}{\left(\frac{\rho_{j-1}}{\rho_{0}}\right)^{7 / 3}-\left(\frac{\rho_{j+1}}{\rho_{0}}\right)^{7 / 3}}\right\},
\end{aligned}
$$

where $p_{0}$ and $\rho_{0}$ are the bulk values. At the first solid layer, $p_{2}=13$ atm and $p_{j \geq 3}=$ svp, we obtain $b_{1} \approx 0.54$ for $\mathrm{Au}$ and $b_{1} \approx 0.58$ for $\mathrm{Cu}$. For both metals, $b_{2} \approx 0.71$, $b_{3} \approx 0.3$, and $b_{j \geq 4}$ are small and form $b_{3 D}$ (the bulk signal). This yields $b_{2 D} \approx 1.55$ for $\mathrm{Au}$ and $b_{2 D} \approx 1.6$ for $\mathrm{Cu}$. As $p_{2}$ increases from 0 to 20 atm, the value of $b_{2 D}$ increases weakly, approximately by $10 \%$. We note that, due to high pressure in the first layers, $\triangle p$ in them can be smaller than the bulk value, but this circumstance does not change the order of $b_{2 D}$, according to estimates.

We can estimate the bulk signal at the electrode with the coordinate $z=0$ in a wave of second sound by using equations (38) and (40) from Ref. 7 (another bulk signal (42), from the DL on the resonator surface, is weaker by one order of magnitude and is omitted). Using $\alpha \Delta T \approx$ $-\triangle \rho / \rho$, we obtain

$$
b_{3 D} \approx \frac{7 \pi S_{7} \gamma\left(R_{r} / L_{r}\right)|e| d_{0}}{6 \varepsilon \bar{R}_{0}^{2} u_{1}^{2}\left(p_{0}\right) m_{4}} \approx 0.48 \gamma\left(R_{r} / L_{r}\right) .
$$

By introducing the potential $\varphi_{\mathrm{h} 0}=-4 \pi d_{\mathrm{h}}\left(\rho_{0}\right) /\left(\varepsilon \bar{R}_{0}^{2}\right)$, we can represent $b_{3 D}$ in the form $b_{3 D} \approx \gamma\left(R_{r} / L_{r}\right) \times$ $7|e| \varphi_{\mathrm{h} 0}\left[6 u_{1}^{2}\left(p_{0}\right) m_{4}\right]^{-1}$ which is similar to the expressions for $b_{2 D}$. The factor $\gamma_{b}\left(R_{r} / L_{r}\right)$ describes the cutting of a bulk signal. The value of such $\gamma$ is determined in Ref. 6: for short and long resonators from the experiment ${ }^{1}$, one has $\gamma \approx 1.4$ and $\gamma \approx 0.05$, respectively. Then $b_{3 D} \approx$ 0.66 and $b_{3 D} \approx 0.024$, respectively. The total values are $b=b_{2 D}+b_{3 D} \approx 2.2$ and 1.57 , respectively, for $\mathrm{Au}$, and $b \approx 2.26$ and 1.62 , respectively, for $\mathrm{Cu}$. The sign of $b$ determines the signal polarity.

First sound was studied $\underline{1}$ for a short resonator; therefore, the signal at the electrode with $z=0$ can be described by (59) at $b \approx 2.2$. The problem consists in the estimation of $\triangle p$. In experiments, $\Delta p$ was not measured. We know ${ }^{47}$ only the maximum power of an acoustic emitter $\left(w_{1 \mathrm{em}} \approx 5 \mathrm{~mW}\right)$ and the $Q$-factor for this power $\left(Q_{1} \simeq 40\right)$.

Firstly, let us consider the experiment with second sound for which the value $\triangle T_{0}$ was measured and let us evaluate this quantity theoretically. This can be done in the usual way, by equating the energy of a temperature wave

$$
\triangle E \approx \frac{\partial E}{\partial T}\langle\triangle T\rangle+\frac{\partial E}{\partial p}\langle\triangle p\rangle
$$


to the pumping energy multiplied by the $Q$-factor: $\zeta w_{\mathrm{em}} \tau Q$. Here, the average is over time and $z$, the quantity $\zeta$ is the coefficient of attenuation (the ratio of the emitted power to the total power of a heat emitter), and $\tau$ is the wave period. It is worth noting that, while considering the energy balance for a standing wave of first or second sound, $T$ and $p$ should be determined from the minimum values in the wave. The energy in a resonator only flows from one side to another side. Its value is not changed, and the losses are exactly compensated by the pumping. Just this energy can be equated to $\zeta w_{\mathrm{em}} \tau Q$. But if $T$ and $p$ are taken from the averaged values, as it is usually done ${ }^{31,44}$, then the main part of the energy (linear approximation) is lost, because it is nullified. Therefore, we consider below $\triangle T(t, z)=0.5 \triangle T_{0}\left[1-\cos \left(\omega_{j} t\right) \cos \left(z \pi / L_{r}\right)\right]$ (and similarly for $p$ ), where $j$ is the sound number.

Using the equations 31,44

$$
\begin{gathered}
E=E_{0}+\mathbf{v}_{s} \mathbf{j}_{0}+\rho \mathbf{v}_{s}^{2} / 2, \quad \mathbf{j}_{0}=\rho_{n}\left(\mathbf{v}_{n}-\mathbf{v}_{s}\right), \\
d E_{0}=T d S+\mu d \rho+\left(\mathbf{v}_{n}-\mathbf{v}_{s}\right) d \mathbf{j}_{0}
\end{gathered}
$$

( $E, S$, and $C$ are given per unit volume) and the thermodynamic relations, we obtain

$$
\triangle E \approx\left(C_{p}-\mu \rho \alpha\right)\langle\triangle T\rangle+\left(\frac{\mu}{c_{1}^{2}}-T \alpha+\frac{T S}{\rho c_{1}^{2}}\right)\langle\triangle p\rangle .
$$

The chemical potential $\mu$ can be determined from the equations

$$
\begin{aligned}
& \mu=\left.\left.\frac{\partial F_{0}}{\partial \rho}\right|_{T} \approx c_{1}^{2} \frac{\partial F_{0}}{\partial p}\right|_{T}= \\
&=\left.c_{1}^{2} \frac{\partial}{\partial p}\right|_{T}\left[E_{0}(T=0)-k_{B} T N_{\mathrm{r}}-\frac{\pi^{4}}{108} k_{B} T N_{\mathrm{ph}}\right]= \\
&=\mu(T=0)+\Delta \mu(T), \\
& \mu(T=0)=\left.c_{1}^{2} \frac{\partial E_{0}(T=0)}{\partial p}\right|_{T} \approx \frac{\epsilon_{0}}{m_{4}} \approx-0.27 c_{1}^{2}, \\
& \triangle \mu(T) \approx-c_{1}^{2} \cdot 10^{-3}\left(1.7\left(\frac{T}{1.6 K}\right)^{4}+\right. \\
&\left.+2.9 \sqrt{\frac{T}{1.6 K}} \exp \left[-\frac{\Delta(T)}{T}+\frac{\Delta(1.6 K)}{1.6 K}\right]\right)
\end{aligned}
$$

Here $\Delta(1.6 \mathrm{~K}) \approx 8.42 \mathrm{~K}$, and $\epsilon_{0}=-7.16 \mathrm{~K}$ is the groundstate energy per atom. In Eq. (70) we used the data 38,48 on the dependence of the parameters of phonons and rotons on $p$.

For second sound, $\Delta \rho \approx-\alpha \rho \Delta T$, and $\Delta p$ is small. Equating energy (67) to the pumping one, we get

$$
\left(C_{p}-2 \mu \rho \alpha\right) \triangle T_{0} / 2 \approx \zeta_{2} w_{2 \mathrm{em}} \tau_{2} Q_{2} / \Omega
$$

where $\tau_{2}=2 L_{r} / c_{2}$, and the factor $1 / 2$ appears on the left-hand side due to the averaging: $\langle\triangle T(t, z)\rangle=\frac{1}{2} \triangle T_{0}$. The linear dependence between $w_{2 \mathrm{em}}$ and $\Delta T_{0}$ was observed in experiments up to the critical value of the heat flow $w_{2 \mathrm{em}, \mathrm{c}} Q_{2} / S_{h}=4 \mathrm{~W} \mathrm{~cm}^{-2}$ at $Q_{2} \simeq 2000$; here $S_{h}$ is the area of the heater. Whence we obtain $\triangle T_{0} \simeq 0.037 \zeta_{2} \mathrm{~K}$ for a short resonator (with the volume $\left.\Omega=L_{r} S_{r} \approx 0.82 \mathrm{~mm}^{3}\right)$ at $T=1.6 \mathrm{~K}\left(S_{r}\right.$ is the area of the resonator, $S_{r} \approx S_{h}$ ). The value of $\zeta_{2}$ is unknown. A wave of second sound includes a small admixture of first sound [in (67), the acoustic wave energy $\sim 4 \%$ at $T=1.6 \mathrm{~K}$ ]. Both sounds are coupled by the relation $\triangle \rho \approx-\triangle T \alpha \rho$. The pumping is only a heat one; therefore $\zeta_{2}$ is determined by the heat inertia ${ }^{49}$ caused by the finiteness of both the heat passage duration through a heater and the durations of the creation and the diffusion of a roton and a phonon (a roton needs a time to depart from the wall and to liberate the place for the next one). In addition, it should be taken into account that, in the presence of a heat pumping, the Kapitsa jump of $T$ arises in the nearsurface layer of He II (with the thickness $\lesssim 10^{-3} \mathrm{~cm}$ ) near a heater 36,37 ,

$$
\triangle T_{\mathrm{K}} \approx \frac{A w}{T^{3} S_{h}}
$$

where $w$ is the power of the heater. For copper, $A \approx$ $5-50 \mathrm{~K}^{4} \mathrm{~cm}^{2} / \mathrm{W}$ (see Ref. 50), and the values of $A$ are close for the other metals. Therefore, at $T=1.4 \mathrm{~K}$ and $w_{2 \mathrm{em}} / S_{h} \lesssim 2 \mathrm{~mW} / \mathrm{cm}^{2}$, we have $\Delta T_{K} \approx 3.6-36 \mathrm{mK}$. Near $T_{\lambda}$, the law (72) is violated. In waves of second sound, we observe two types of oscillations of $T$ : bulk ones and oscillations in the near-surface layer [due to the jump (72)]. At the resonance, these oscillations must be consistent. Since $\Delta T_{\mathrm{K}}$ sets the amplitude of variations of $T$, the relation $\triangle T_{0} \lesssim \triangle T_{\mathrm{K}}$ should be valid. At the resonance, we may expect that $\Delta T_{0} \sim \triangle T_{\mathrm{K}}$ (in the ideal system, where the heater, walls, and the thermometer are made of the same material, it is probable that $\triangle T_{0}=$ $\left.\triangle T_{\mathrm{K}}\right)$. From the experiment, we have $\Delta T_{0} \simeq 3 \mathrm{mK}$ at $T=1.4 \mathrm{~K}$, and $\Delta T_{0} \simeq 1.3 \mathrm{mK}$ at $T=1.6 \mathrm{~K}$. One can see that, at $T=1.4 \mathrm{~K}$, the quantity $\triangle T_{0}$ is of the same order as $\triangle T_{\mathrm{K}}$, being several times smaller. More exact data of a new experiment ${ }^{47}$ indicate that their dependencies on the temperature are also close. The experimental value of $\triangle T_{0}$ at $T=1.6 \mathrm{~K}$ is obtained at $\zeta_{2} \simeq 1 / 29$. It is apparent that $\zeta_{2}$ is determined by the value of $\Delta T_{\mathrm{K}}$, i.e., by the Kapitsa jump.

We note that formula (71) without both $\zeta_{2}$ and a correction with $\mu$ was obtained earlier in Ref. 51 in a different way. In Ref. 49, it was observed that the attenuation is almost absent $\left(\zeta_{2} \simeq 1\right)$ for second sound at frequencies $\nu \sim 100-500 \mathrm{~Hz}$. However, strong attenuation occurs at $\nu \gtrsim 5 \mathrm{kHz}$, which corresponds to the result obtained above: $\zeta_{2} \approx 1 / 29$ for $\nu \approx 10 \mathrm{kHz}$.

Let us consider first sound. From the system of equations for first and second sounds (see Ref. 31, Chap. 1, $\S 7)$, it is easy to obtain that

$$
\triangle T \approx-\triangle p \cdot \alpha T / C_{p} .
$$


Using Eqs. (67)-(70) and (73) and equating $\triangle E$ to the pumping $\zeta_{1} w_{1 \mathrm{em}} \tau_{1} Q_{1}$ with $\tau_{1}=2 L_{r} / c_{1}$, we get

$$
\frac{\Delta p_{0}}{2}\left(\frac{\mu}{c_{1}^{2}}-2 T \alpha+\frac{T S}{\rho c_{1}^{2}}+\frac{\mu \rho T \alpha^{2}}{C_{p}}\right) \approx p_{1 E}
$$

where $p_{1 E}=\zeta_{1} w_{1 \mathrm{em}} \tau_{1} Q_{1} / \Omega$. The quantity $\zeta_{1}$ and the critical flow $w_{1 \mathrm{em}, \mathrm{c}} Q_{1} / S_{h}$ are unknown, and the analysis performed for second sound indicates that they involve a sufficiently complicated physics. We only note that an acoustic emitter was the membrane of a headphone, in which the electric signal $w_{1 \mathrm{em}}$ is transformed into an acoustic one only partially. In addition, the frequency of first sound is larger by a factor of 11.5 than that of second sound, which favors a decrease of $\zeta_{1}$. For estimates, let us take $\zeta_{1}=\zeta_{2}=1 / 29$ and $w_{1 \mathrm{em}, \mathrm{c}} Q_{1} / S_{h}=$ $w_{2 \mathrm{em}, \mathrm{c}} Q_{2} / S_{h}=4 \mathrm{~W} \mathrm{~cm}^{-2}$. Then $p_{1 E} \approx \zeta_{1} \cdot 3.4 \cdot 10^{-3} \mathrm{~atm}$ and $\Delta p_{0} \approx-0.026 \zeta_{1} \mathrm{~atm} \approx-9 \cdot 10^{-4} \mathrm{~atm}$ (the minus sign indicates that the internal energy decreases in the region of enhanced pressures, and we will use $\left|\Delta p_{0}\right|$ in what follows). In this case, the pressure of saturated vapor is $p_{0} \sim 2.5 \cdot\left(10^{-3}-10^{-2}\right) \mathrm{atm}$ at $T=1.4-1.8 \mathrm{~K}$. With regard for (59) at $b \approx 2.2$, we obtain a signal $\triangle \varphi(z=0) \approx 57 \mathrm{nV}$, which is about the minimally registered signal $10 \mathrm{nV}$ by the order of magnitude. Apparently, a significantly smaller $\Delta p_{0}$, less than $10^{-4}$ atm, was attained in experiments, and $\Delta \varphi$ turned out to be below the threshold of registration.

It is interesting that $\sim 97 \%$ of the energy of acoustic oscillations [the second term in [67]) are given by the term $\mu(T=0)\langle\Delta p\rangle / c_{1}^{2} \approx-0.3\langle\Delta p\rangle$ which is the energy of pulsations of the ground state of He II. These pulsations are running together with the movement of quasiparticles, but the energy related to quasiparticles is smaller by two orders of magnitude. Thus, the negative energy of the "vacuum" dominates.

We note that, according to (67), a standing half-wave of first sound is always accompanied by a low-intensity wave of second sound (heat oscillations) bearing $\sim 1.5 \%$ of the energy. However, heat oscillations are easily absorbed by the walls, so that the heat losses are significantly greater than $1.5 \%$. In this case, the heat pumping is absent, and the transformation of the acoustic energy to quasiparticles is slow. Therefore, a wave of second sound gives possibly the main contribution to the attenuation of first sound. If this is true, then $\Delta p_{0}$ can be increased by two measures: by completely suppressing a heat wave (to the level of fluctuations) or, on the contrary, by its pumping. In this case, the $Q$-factor must sharply increase. The first measure can be realized by approaching the temperature at which $\alpha \approx 0(T \approx 1.12 \mathrm{~K}$ or $1.18 \mathrm{~K}$, according to Refs. 38 and 31 , respectively). In the second case, it is necessary to induce a heat pumping (with the frequency and phase of first sound) in addition to the acoustic one. One more measure consists in the maximum decrease of the frequency with increase in the resonator length. Finally, it is possible to use the method of filtration for the generation of first sound 49,51 . According to Ref. 49, this method allows one to attain $\triangle T_{1} \simeq \triangle T_{2} / 6$ and to obtain large $\Delta p_{0}$ up to $0.1 \mathrm{~atm}$. In these case, the signal $\Delta \varphi$ should be strong (up to $1000 \mathrm{nV}$ ) and observable.

It is worth noting that the absence of any information about $\zeta_{1}$ and $w_{1 \mathrm{em}, \mathrm{c}} Q_{1} / S_{h}$ requires to measure the value of $\Delta p_{0}$ directly or to measure $\Delta T_{0}$ and then to determine $\triangle p_{0}$ from (73).

We believe that the electric signal arises in a wave of second sound because such a wave is always accompanied by first sound. In this case, second sound is only a way to generate first sound, and, under certain conditions, this way is more efficient than the direct acoustic generation of first sound. Undoubtedly, there exist methods of direct excitation of first sound which allow one to obtain highamplitude pressure oscillations and a large signal $\Delta \varphi$. Due to the coupling of the two sounds, the nature of the electric signal can be understood only by simultaneously studying both sounds. Therefore, the further studies of first sound appear to be of great importance.

\section{DISCUSSION OF THE RESULTS AND A NEW EXPERIMENT}

We note some specific features of the electric signals for first and second sounds. According to the model, the signal has the same nature for both sounds and is related to oscillations of the density. The difference consists in the following: for first sound, oscillations of the density are induced directly, whereas for second sound they are generated indirectly due to the weak coupling of the two sounds. For both sounds, the signal consists of the surface and bulk parts. The first part dominates and does not depend on the size of the resonator and on the temperature. Moreover, the signal for first sound does not depend on $T$ irrespective of the consideration of the exhaustion of $\rho_{s}$ at the wall, whereas it is necessary to take the behavior of $\rho_{s}$ into account for second sound. It is also clear that the signal for first sound is not related to superfluidity and must be observed above $T_{\lambda}$. For second sound, some limitation consists in that the connection between $\nabla T$ and $\nabla \rho$ is unclear above $T_{\lambda}$. Moreover, the quasiparticles are poorly defined above $T_{\lambda}$, so that second sound cannot be excited. Nevertheless, the effect should be observable above $T_{\lambda}$ if stable oscillations of the temperature can be induced and if $\nabla T$ generates $\nabla \rho$.

It is interesting that the constants $a$ and $b$ are close to unity in the dimensional formulas for both effects. This is apparently related to the dominance of the surface polarization induced in several atomic layers: the constants $a$ and $b$ must be of the order of unity if the effect arises on atomic scales.

In addition, the theory predicts a number of peculiarities. Namely, for both sounds, the signal $\triangle U$ must significantly differ for electrodes belonging to different groups: those forming two solid layers of helium, those forming one solid layer, those that do not form solid layer, but are wetted, and those that are nonwetted. In this case, 
the signals must be close for electrodes made of different metals of the same group, although the binding energies of the ${ }^{4} \mathrm{He}$ atom are significantly different for different metals of the group. Such a closeness of signals is mainly related to the fact that the metal-induced polarization of a ${ }^{4}$ He atom located near the electrode gives a significantly smaller contribution to the signal than the polarization which is induced by the half-space with helium and does not depend on the kind of a metal. This can be verified in experiments. The three electrodes from the experiment 1 probably belonged to the same group. Therefore, the signals were practically identical for them. Additional differences can be for metals with the hexagonal lattice and for intermetals with a number of unusual properties, including a large coefficient $\alpha$ of thermal expansion approaching that for He II.

The coefficients $a$ and $b$ are calculated with an error of about one order of magnitude. It is caused by the error of the correlation function $g(r)$ at small $r$ and by the neglect of anisotropy of $g(\mathbf{r})$ near the surface, as well as by the neglect of the difference between the longitudinal and transverse coefficients of linear expansion for the first layers. This leads to the error of $a_{j}$ and $b_{j}$ up to several times. A considerable error for $a=a_{1}+a_{2}+a_{3}$ is introduced by the difference of signs of $a_{1}$ and $a_{3}$ which are almost identical in absolute value. The summary error of $a$ is about one order of magnitude. For $b$, all components $b_{j}$ are of the same sign and order. The quantities $a_{j}$ are also sensitive to both the value of $p_{2}$ and the distribution of $T$ in the first layers, whereas $b_{j}$ are insensitive to them. Therefore, $b$ can be calculated more reliably than $a$ with an error up to several times.

In calculations, we did not consider the microroughness of the metal surface and the oxide film. The microroughness should not be significant since the value of $d_{\text {mir }}$ must be the same for different charge distributions in the metal and equal to $d_{\text {mir }}$ from the exact mirror image. But an oxide film can significantly change the results, and this case must be examined specially. Some electrodes are easily oxidized in air, and their surface should be specially prepared to prevent the formation of an oxide film.

We also did not take into account the inertial polarization of ${ }^{4} \mathrm{He}$ atoms arising due to their collisions with the metal surface.

We were interested only in metals with a cubic lattice. However, a lot of metals has a hexagonal lattice, for which the Wigner-Seitz cell has a quadrupole moment creating an electric field. Under the action of this field, the conduction electrons are redistributed in a metal so that the field inside and outside the metal become zero due to the formation of a dipole layer on the metal surface. This dipole layer will additionally contribute to $d_{1}$ (the DM of ${ }^{4} \mathrm{He}$ atom at the metal surface). The induced DM is very large, $\sim 1000 d_{\text {mir }}$, but it is compensated by DMs from the quadrupoles of cells. However, due to the exchange interaction, the compensation is not complete, which can considerably change $d_{1}$ and $a$.

Let us consider the question about a jump of $T$ of he- lium in several near-surface layers. The existence of such a jump follows from the reasoning of Sec. III and allows us to explain the $T$-independence of the signal $U$ in the experiment with second sound. As was mentioned above, a jump of $T$ in near-surface layers of helium was discovered by Kapitsa ${ }^{36}$ and is related to the high heat conductivity of helium. But the mechanism considered in Sec. III predicts a jump of $T$ in a thinner layer $\sim 10 \AA$ at the wall. This jump has a different nature and is related to the condensation of rotons on the wall. This mechanism seems to be important, because it concerns also the other properties such as the exhaustion of $\rho_{s}$, dry friction, and $T$-independence of $U$. This question deserves a separate more strict study. The key assumption of our consideration is that the condition $\rho_{s}=0$ on the wall yields the equality $T=T_{\lambda}$ on the wall (see Sec. III). This allows us to conclude that, in the first layers at the wall, $T$ must smoothly vary from the bulk temperature far from the wall to a higher $T$ of the wall equal to $T_{\lambda}$ at the pressure on the wall. The other reasonings lead to the same conclusion. Rotons at the wall have energy that is less by $2 \mathrm{~K}$ than those of bulk rotons. Hence, their concentration is higher by several times. However, these 2D-rotons involve atoms of several layers nearest to the wall into the motion. Hence, the temperature of the medium at the distance of 2-3 atomic layers from the wall is determined not only by bulk quasiparticles, but by surface ones as well. But the number of 2Drotons is greater by several times than that of bulk ones. Therefore, 2D-rotons significantly increase the temperature of the first layers. In addition, we should expect the presence of the exchange by quasiparticles between the wall and the bulk. Since the energy and the concentration of 2D-rotons and phonons differ from their values for bulk quasiparticles, 3D-rotons and phonons near the wall undergo the different actions from the sides of helium (3D-rotons and phonons) and the wall (2D-rotons, 2D-phonons, and atoms of the wall). In other words, the equilibrium concentration of 3D-quasiparticles at the wall must differ from the concentration far from the wall. Hence, we have $\nabla_{z} T \neq 0$ near the wall. The thickness of the layer with $\nabla_{z} T \neq 0$ is $\sim$ the effective size of a roton ( $\sim 3$ atomic layers). This is in agreement with the experimental data on the third-sound ${ }^{30,52}$ which give the thickness of the layer with $\rho_{s} \approx 0$ equal to $2 \div 4$ at. layers at $T=1.4 \div 1.8 \mathrm{~K}$. From the viewpoint based on the symmetry, the gradient of $T$, like the gradients of pressure and polarization, is related to the system isotropy breaking at the wall.

At the present time, a new experiment is carried on with second sound ${ }^{47}$ to determine the dependence of the electric signal on the coordinate $(Z)$ along a resonator and the temperature. We will determine the possible results of the experiment within the present model.

Since the model considers the signal as mainly the surface one, the signal dependence on $z$ must be completely determined by the dependence (16) of the temperature of He II on $z$. Above, we have determined the potential $\varphi$ 
at the end electrode $(z=0)$. In the experiment, the potential difference $U$ between two electrodes is measured. The amplitude $\Delta U$ of the potential difference between the electrode with coordinate $z$ and the ground is given by formula (33), where $a$ depends on $T_{0}$ and surface properties (and is independent of $z$ ), and $\Delta T$ depends on $z$ by (16) as $\Delta T(z)=\Delta T_{0} \cos \left(\pi z / L_{r}\right)$. Whence

$$
\triangle U(z)=a \frac{k_{B}}{2|e|} \triangle T(z)=a \frac{k_{B}}{2|e|} \triangle T_{0} \cos \left(\pi z / L_{r}\right) .
$$

The potential difference between identical ungrounded electrodes with the coordinates $z$ and 0 is equal to

$$
\triangle U(z)=a \frac{k_{B}}{2|e|} \triangle T_{0}\left[1-\cos \left(\pi z / L_{r}\right)\right] .
$$

Two last formulas are true for the surface signal. To make comparison with the experiment, we need to know also the $Z$-dependence of the bulk signal that is registered by a ring electrode positioned on the internal surface of the resonator. The amplitude of the potential difference between this electrode with coordinate $Z$ and the ground follows from formulas (52)-(54) after the replacement $R \rightarrow R_{r}, \cos \left(\omega_{2} t\right) \rightarrow 2$ :

$$
\triangle U_{b}(z)=\varphi_{b}(Z)=2 \varphi_{0}(T) \gamma_{b}\left(Z, R_{r}, \sigma\right) .
$$

The dependence of $\gamma_{b}$ on $Z$ at $R=R_{r}$ for the experimental value $\sigma=0.2$ is given in Fig. 4. In Fig. 5, we present the $Z$-dependencies of the surface (75), bulk (78), and total signals. For the surface signal, we took $a \approx-0.8$ for copper (see Sec. IV).

As seen from Fig. 5 , the dependencies for the surface and bulk signals are somewhat similar, but are different. Their similarity is due to the fact that the main contribution to the bulk potential is given by the region of helium near the electrode. The difference is related to that this region has a macroscopic thickness $\left(\sim 2 R_{r}\right)$, whereas the thickness corresponding to the surface signal is equal to several atomic layers.

At the derivation of the potential difference (78), we did not take into account that the electrodes in the new experiment are covered by a dielectric film. In addition, it is shown in Sec. VI that the bulk signal is completely damped by an admixture of polar molecules, if its partial concentration $\gtrsim 10^{-12}$. Such an admixture is always present, most likely, in helium, which nullifies the bulk signal. In Fig. [5] we did not consider the bulk signal (42) arising due to DL on the electrode surface. Such a signal is analogous to the surface signal by its properties and is less by two order of magnitude by intensity. Therefore, we omit it. In addition, this bulk signal is easily damped by polar admixtures at their concentrations $\gtrsim 10^{-14}$. Moreover, formula (78) does not involve the images of dipoles in a metal. On the resonator walls parallel to the resonator axis, these images-dipoles are directed oppositely to dipoles in helium and, therefore, decrease significantly the potential on ring electrodes (probably by several times). For the electrode on the plane end

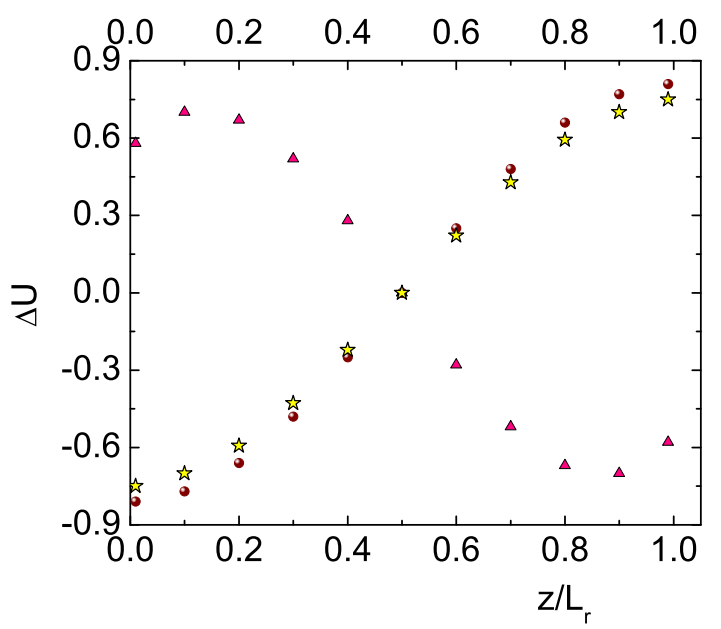

FIG. 5: Theoretical potential difference $\triangle U(z)$ (normalized to $\left.k_{B} / 2|e|\right)$ between a ring electrode with coordinate $z$ and the ground. The symbols $\bullet \bullet-$ show the $z$-dependence for the surface signal (75), and $\triangle \triangle \triangle$ are related to the bulk signal (78) increased by 10 times for clearness. $\star \star \star$ is the summary curve of the bulk and surface signals. The bulk signal is presented under experimental conditions with $\sigma=$ 0.2 and $T=1.8 \mathrm{~K}$, and the damping due to images and admixtures is not taken into account in this signal.

surface, the images-dipoles are directed as the dipoles in helium. Therefore, they increase the signal up to two times.

The curves in Fig. 5 are the prediction of the model. The same dependencies on $Z$ are obviously valid for the surface signal from first sound. The same dependencies on $z$ are obviously valid for the surface signal from first sound.

Since the electrode is covered by a dielectric, the potential on the electrode surface is induced by three sources: the polarized first layers of helium on the dielectric surface and layers of a dielectric on the surfaces of helium and a metal. We will determine the electrode potential analogously to (15). With regard for only the first layers, we obtain

$$
\varphi \approx-\frac{8 \pi d_{1}}{\left(\varepsilon_{\mathrm{He}}+\varepsilon_{\mathrm{d}}\right) \bar{R}_{1 \|}^{2}}-\frac{8 \pi d_{2}}{\left(\varepsilon_{\mathrm{He}}+\varepsilon_{\mathrm{d}}\right) \bar{R}_{2 \|}^{2}}-\frac{4 \pi d_{3}}{\varepsilon_{\mathrm{d}} \bar{R}_{3 \|}^{2}},
$$

where $\varepsilon_{\mathrm{He}}$ and $\varepsilon_{\mathrm{d}} \simeq 3$ (see Ref. 47) are the dielectric permittivities of helium and a dielectric, $d_{1}, d_{2}$, and $d_{3}$ are, respectively, the dipole moments of an atom in the first layer of helium and the first layer (near helium and a metal) of a dielectric, and $\bar{R}_{j||}$ are the mean longitudinal interatomic distances for these layers. For the first and second terms, we used the fact that a charge $q$ located in the first dielectric creates the potential $\varphi_{2}=\frac{2 q}{\left(\varepsilon_{1}+\varepsilon_{2}\right) R}$ in the second dielectric and $\varphi_{1}=\frac{q}{\varepsilon_{1} R}+\frac{\left(\varepsilon_{1}-\varepsilon_{2}\right) q}{\varepsilon_{1}\left(\varepsilon_{1}+\varepsilon_{2}\right) R^{\prime}}$ in the first one (see $\S 23$ in Ref. 39). In our case, $R^{\prime}=R$; therefore, $\varphi_{1}=\frac{2 q}{\left(\varepsilon_{1}+\varepsilon_{2}\right) R}=\varphi_{2}$. 
Let us evaluate $a$. If $\nabla p=0$ in helium near the dielectric surface, then the helium-related part $a$ is determined by formulas of Sec. IV B. But if $\nabla p \neq 0$, we take formulas of Sec. IV A. The contribution to $a$ from the layers of dielectric atoms consists of the following parts: one proportional to $\alpha^{s}$ of the dielectric (which must be small, since $\alpha^{s}$ of solids is usually much less than that of liquid helium) and one proportional to $\alpha$ of helium. The latter should be comparable with $a$ determined for helium in Sec. IV A or B. This implies that $a$ should be of the order of magnitude of $a \approx-1$ from the first experiment $\mathrm{t}^{\underline{1}}$, but no exact coincidence is expected.

Recently, we have read Ref $\underline{11}$, where a bulk model of polarization is proposed. Figure 2 of that work shows the experimental dependence of the signal in a second-sound wave on the coordinate $Z$ along a resonator. Though the experimental data have not been published yet, we will make a preliminary comment. The points indicated in Ref. 11 agree with formula (75) describing the surface signal. The curve for the bulk signal differs from the experimental one by shape. The summary curve (Fig. 5) representing the surface and bulk signals does not differ by shape from the purely surface curve due to the smallness of the bulk signal. Therefore, it is difficult to separate the bulk signal by the experimental points given in Ref. 11. However, the bulk contribution increases strongly, as the temperature increases and the ratio of the resonator length to its radius decreases. With regard for this fact, it would be possible to determine the bulk signal, by using the full experimental data, when they will be published. The data available at present indicate the mainly surface nature of the signal.

\section{CONCLUSIONS}

We have approximately calculated the electric signal $U$ arising at the electrode in the presence of a standing half-wave of first or second sound in He II. The properties of the signal for second sound correspond to the experiment in its amplitude (approximately) and its independence on the resonator size and the temperature. Therefore, we have impression that the nature of the signal is clear on the whole. However, the properties of He II near the metal surface are not clear in some aspects and are described only approximately. Therefore, the subsequent studies should be focused on this region. The signal for first sound has not been discovered experimentally till now, so that the formulas concerning first sound are the predictions of the model. In addition, the model predicts the formula of the dependence of the signal on the coordinate $z$ along a resonator and the strong growth of the signal for ${ }^{3} \mathrm{He}-{ }^{4} \mathrm{He}$ mixture. It is shown also that the insignificant random admixtures $\left(\gtrsim 10^{-12}\right)$ of polar molecules can completely damp the bulk polarization of helium in first- and second-sound waves almost not affecting the surface polarization. Therefore, we risk to assume that the bulk polarization is possible only in He II specially purified from admixtures.

In this connection, we indicate the problems of dry friction 27 and the exhaustion of $\rho_{s}$ at the wall. The consideration in Sec. III implies that the dry friction can appear below $T_{c} \sim 0.7 \mathrm{~K}$. It should be of interest to measure it at all temperatures from 0 to $T_{\lambda}$.

To summarize, further studies are needed to attain complete understanding of the nature of the effect. In particular, is importantly to test experimentally the predictions of different models to determine the correct one.

\section{Acknowledgments}

The author is grateful to A. B. Kashuba, V. M. Loktev and Yu. V. Shtanov for useful discussions and to A. S. Rybalko for his comments and some preliminary information about the new experiment. The author also thanks M. M. Bogdan, E. Ya. Rudavskii and the participants of the seminar at the Verkin Institute for Low Temperature Physics and Engineering for critical discussion of the results.

\section{Appendix A. Deja vu: the electric field in a spontaneously polarized dielectric}

We now discuss the methods of calculation of the electric field in a "spontaneously" polarized dielectric such as He II with first- or second-sound waves. In the literature, two different methods leading to completely different results are developed. Therefore, we will consider this question in more details.

In a number of works, in particular in one of the first works ${ }^{4}$ and in new works ${ }^{11,12}$, the bulk models of the electric activity of He II are considered without regard for the surface polarization. In Refs. 4, 11, the polarization of helium is explained by that the atoms in a second-sound wave are accelerated and are polarized due to the inertia. The coupling of the polarization and the acceleration $\mathbf{w}$ is described by the Melnikovsky formula

$$
\mathbf{P}=-\gamma_{i} \mathbf{w} .
$$

According to Ref. 12, the polarization is related to collisions of atoms; as a result, formula (79) acquires the different coefficient. In these works, the potential is calculated by the formula

$$
\mathbf{E}=-\nabla \varphi,
$$

and the field strength $\mathbf{E}$ is determined by two means. In Ref. 11, it is described by the formula

$$
\mathbf{D}=\varepsilon \mathbf{E}, \quad \mathbf{P}=\kappa \mathbf{E},
$$

where $\kappa=(\varepsilon-1) / 4 \pi$ is the polarizability. In Refs. 4 and 12 , the field strength is determined from the condition

$$
\mathbf{D}=0, \quad \mathbf{E}=-4 \pi \mathbf{P} .
$$


The proportionality $\mathbf{E}=$ const $\times \mathbf{P}$ allows one to obtain a bulk electric signal that is of the order of the experimental one and does not depend on the resonator size. However, we do not agree with some positions of these works, in particular with the formula $\mathbf{w}=\partial\left(\mathbf{v}_{n}-\mathbf{v}_{s}\right) / \partial t$ for the acceleration ${ }^{11.12}$ and with the method of estimation of the coefficient $\gamma_{i}$ in formula (79). The relation $\mathbf{w}=\partial\left(\mathbf{v}_{n}-\mathbf{v}_{s}\right) / \partial t$ is an assumption, it allows one to obtain a signal weakly depending on the temperature. By its physical sense, the quantity $\mathbf{w}$ in formula (79) is the local acceleration of a medium. It is described by the formula $\mathbf{w}=\frac{D}{D t} \frac{\rho_{n} \mathbf{v}_{n}+\rho_{s} \mathbf{v}_{s}}{\rho}$ that is valid for first and second sounds. The coefficient $\gamma_{i}$ was calculated in Refs. 11,12 in the first order of the stationary perturbation theory. But, even in the calculation of the mutual (tidal) polarization of two immovable atoms, the second order of perturbation theory is used ${ }^{14-16}$. The polarization arises at the acceleration due to the interaction of atoms. Therefore, it is one of the manifestations of the tidal polarization of atoms. Respectively, it can be calculated for two atoms analogously to Refs. 14 16 in the second order of perturbation theory, but nonstationary. Hence we can estimate also the value of $\gamma_{i}$ for the medium. Such a procedure of calculation of $\gamma_{i}$ seems more exact.

We now consider the methods of calculation of the electric field for the given problem in more details, since this question is of principal meaning. We have He II with a second-sound wave in the case where the polarization of a medium (dielectric) is related to internal processes in the fluid, and no external electromagnetic fields are present. Such a statement of the problem is common for all authors. The key point is that without external field $\mathbf{E}$ the source of the field $\mathbf{E}$ is the polarization of atoms. The problem can be solved within two exact approaches: 1) to solve the Maxwell equations

$$
\operatorname{div} \mathbf{D}=0, \quad \operatorname{rot} \mathbf{E}=0
$$

with regard for boundary conditions or 2) to calculate the potential as a sum $\varphi(\mathbf{R})=\sum_{j} \frac{e_{j}}{\left|\mathbf{R}-\mathbf{r}_{j}\right|}$ of the potentials from all charges of the system (electrons and nuclei of the helium atoms). The helium atoms are not charged on the whole. Therefore, the potential is reduced to the sum of the potentials from dipoles and higher multipoles of atoms. Since the contribution of dipoles is usually dominant, it is sufficiently to sum over them:

$$
\varphi(\mathbf{R})=n \int d \mathbf{r} \frac{\mathbf{d}(\mathbf{R}-\mathbf{r})}{\varepsilon|\mathbf{R}-\mathbf{r}|^{3}} .
$$

Here, $\mathbf{r}$ are the coordinates of atoms of helium, and $\mathbf{R}$ is the observation point. Respectively, the field strength

$$
\mathbf{E}(\mathbf{R})=-\nabla_{\mathbf{R}} \varphi=\int d \mathbf{r} \frac{3(\mathbf{P n}) \mathbf{n}-\mathbf{P}}{|\mathbf{R}-\mathbf{r}|^{3}},
$$

where $\mathbf{P}=n \mathbf{d} / \varepsilon$ is the polarization and $\mathbf{n}=(\mathbf{R}-\mathbf{r}) / \mid \mathbf{R}-$ $\mathbf{r} \mid$. The full polarization consists of spontaneous and induced parts:

$$
\mathbf{P}=\mathbf{P}_{s p}+\mathbf{P}_{i n d}, \quad \mathbf{P}_{i n d}=\kappa \mathbf{E} .
$$

In view of the smallness of $\kappa$ for He II, the induced part is very small and can be neglected, if the spontaneous polarization is the primary source of the field. Such a method was used in Refs. 6 - 9 and above.

In Refs. 4, 11, 12, the calculation is performed in a different way with the use of relations (81) or (82). The results turn out quite different. In particular, it was shown ${ }^{6.7}$ that the bulk signal depends strongly on the resonator size. But no such dependence was found in Refs. 4, 11, 12 and in experiments. Therefore, to clarify the nature of the signal, it is important to know which method is more exact.

First, let us consider the applicability of the condition $\mathbf{D}=0, \mathbf{E}=-4 \pi \mathbf{P}$ in the bulk of helium. As is known, this condition is valid on the boundary of a dielectric and a metal. In the bulk, it is true only in several cases. If a dielectric is surrounded by a resonator positioned in an external field $\mathbf{E}$, then the condition $\mathbf{D}=0$ is satisfied in the bulk in the absence of the spontaneous polarization in a dielectric. In this case, we have also $\mathbf{E}=-4 \pi \mathbf{P}=0$. In our problem, the external field is absent, and the polarization of helium is spontaneous. Moreover, the condition $\mathbf{D}=$ const can be satisfied (which gives the relation $\mathbf{E}=-4 \pi \mathbf{P}$ for the variable field), if a dielectric is homogeneous in the direction of the vector $\mathbf{D}$. In our case, the situation is different, and the polarization is caused namely by the inhomogeneity. This implies that the condition $\mathbf{D}=0$ is not satisfied in the bulk for our problem. For the verification, we use formula (85) to determine divE with regard for the relation $\triangle_{\mathbf{R}}|\mathbf{R}-\mathbf{r}|^{-1}=-4 \pi \delta(\mathbf{R}-\mathbf{r})$. We obtain $\operatorname{div} \mathbf{D}=0$, i.e., the Maxwell equation is satisfied. Analogously, we determine $\mathbf{E}$, by assuming for simplicity that the polarization $\mathbf{P}$ is directed along the $Z$ axis (as in the experiment). We obtain

$$
\begin{gathered}
\mathbf{E}=-4 \pi \mathbf{P}+\tilde{E} \mathbf{i}_{z}+\mathbf{i}_{x} \int d \mathbf{r} \frac{P(\mathbf{r}) \partial^{2}}{\partial R_{x} \partial R_{z}} \frac{1}{|\mathbf{R}-\mathbf{r}|}+(x \leftrightarrow y), \\
\tilde{E}=\int d \mathbf{r} P(\mathbf{r})\left(\frac{\partial^{2}}{\partial R_{z}^{2}}-\triangle_{\mathbf{R}}\right) \frac{1}{|\mathbf{R}-\mathbf{r}|} .
\end{gathered}
$$

As is seen, the field is more complicated than that by the relation $\mathbf{E}=-4 \pi \mathbf{P}$. Indeed, we can separate the component $-4 \pi \mathbf{P}$ from $\mathbf{E}$. But, in addition, we have nonzero components along the other axes and the component $\tilde{E}$ along the $Z$ axis. The numerical analysis indicates (see Sec. VI) that last component is great. If we set $\mathbf{E}=\nu \mathbf{P}$, then the coefficients of proportionality $\nu$ differ significantly from one another at different points and depend on the resonator size. For example, at $R_{r} / L_{r}=0.2$ and $T=1.8 \mathrm{~K}$, we have $\nu \approx-2.5$ at the point in the middle of the resonator axis $\left(Z=L_{r} / 2\right)$, and $\nu \approx 1 / \kappa \approx 220$ at the beginning of the axis $(Z \rightarrow 0)$. Near the surface of the electrode, it is necessary to consider the presence of several strongly polarized layers of helium. In this case, the field is perpendicular to the surface, the condition $\mathbf{D}=0$ is satisfied, and it yields potential (15), (30), (32). 
In Ref. 11, the field $\mathbf{E}$ is determined from formulas (81). However, these formulas are valid only in the case where a dielectric is polarized by an external field. Indeed, the relation $\mathbf{P}=\kappa \mathbf{E}$ expresses the fact that the electron shells of atoms of a dielectric are stretched in an external field $\mathbf{E}$, i.e., this relation describes a response of the medium to an external field. In our problem, no external field is present, and the source of the field $\mathbf{E}$ is the polarization of helium atoms. This polarization is related to their interaction. We consider that the main contribution to the polarization is given by the tidal polarization of atoms arising in immovable atoms due to the interaction ${ }^{14-16}$. In Refs. 4, 11, 12, it is considered that the polarization is related to a motion. It has no meaning in the sense that the polarization is caused, in any case, by processes in a fluid and is not connected with an external field. Such a polarization is named spontaneous. It is also observed in piezoelectrics and pyroelectrics. In helium, a different mechanism acts. At the spontaneous polarization, the relation $\mathbf{P}=\kappa \mathbf{E}$ does not hold, obviously.

For clearness, we consider a simple example from the course of electrodynamics 39.53 : let us determine the field inside of a uniformly polarized dielectric ball with radius $R_{0}$. We consider that the polarization $\mathbf{P}$ is the same by direction and by magnitude at all points of the ball. The simple reasoning (see Ref. $39, \S 24$, and Ref. 53, $\S 13$, Exercise 1) allows one to write the answer for the field at a point $\mathbf{R}$ :

$$
\varphi(\mathbf{R})=4 \pi \mathbf{P R} / 3, \quad \mathbf{E}(\mathbf{R})=-4 \pi \mathbf{P} / 3 .
$$

We can verify that the same result follows from the direct summation of the contribution of dipoles according to (84) and (85). As is seen, the solution is obtained correctly in approach (84), but relations (81) and (82) are not satisfied in such a system. We note that, despite the proportionality of $\mathbf{E}$ and $\mathbf{P}$ in (89), the connection between them is nonlocal (as distinct from the local relations (81) and (82)), since the coefficient of proportionality is determined by the contribution of all dipoles of a dielectric from the region $r \leq R$. Qualitatively, the problem for helium in a resonator is analogous. But the polarization in helium is inhomogeneous, a vessel is not sphere-like, and helium is bounded by a resonator; therefore, the connection between $\mathbf{E}$ and $\mathbf{P}$ is more complicated, than (89). To estimate the role of the inhomogeneity, we consider that the polarization of a ball increases proportionally to $r: \mathbf{P}(\mathbf{r})=4 r \mathbf{P}_{0} / 3 R_{0}$ (in this case, the average over the bulk $\langle\mathbf{P}\rangle=\mathbf{P}_{0}$ ). Then the calculation gives

$$
\varphi(\mathbf{R})=\pi \mathbf{P R}, \quad \mathbf{E}(\mathbf{R})=-\pi(\mathbf{P}+\mathbf{n}(\mathbf{n P})),
$$

here $\mathbf{P} \equiv \mathbf{P}(\mathbf{R})$ and $\mathbf{n}=\mathbf{R} / R$. In other words, the inhomogeneity leads to a change of the coefficient and to a complication of the dependence $\mathbf{E}$ on $\mathbf{P}$. If the ball is stretched into a "cigar", then the dependence of the field on the ratio of the sizes of a cigar appears. In the ball, the field from external uniformly polarized layers is equal to zero, since the layers are spherical. But they are nonspherical in the cigar, therefore the field is nonzero, and the dependence on sizes of the system appears. If a dielectric is covered by a metal, the field changes due to the contribution of images in a metal.

We note one more point. In approaches (80)-(82), the potential difference is determined as the integral $\Delta \varphi=-\int$ Eds. In Refs. 4, 11, 12, the contour is chosen along the resonator axis $z$ between points on the resonator ends. In the experiment $\frac{1}{\underline{1}}$, one electrode is positioned on the resonator end, and the second electrode is the metallic resonator itself. In this case, the potential difference between the end electrode and any point of the resonator is the same. But if the potential difference is calculated by the relations $\Delta \varphi=-\int$ Eds and (81) or (82), by positioning the contour beginning on the first electrode and the contour end on the internal surface of the resonator, then $\Delta \varphi$ depends on the coordinate $z$ of a point of the resonator. In particular, for the points on the lateral surface with the coordinates $Z=0,0.5 L_{r}$, and $L_{r}$, the potential difference is equal to 0,1 , and 2 (in arbitrary units). This circumstance indicates that the field $\mathbf{E}$ is determined inaccurately. The equivalence of different contours requires, in particular, that the field have the great $\rho$-component. The approach in (84) has the same difficulty: it follows from formulas (52)-(54) and Fig. 4 that the bulk potential difference between the end electrode and points on the lateral surface of a resonator with $Z=0,0.5 L_{r}$, and $L_{r}$ is equal to $0.16,0.49$, and 0.82 . Such a distinction is related to the neglect of the images of charges in a metal. The images must significantly affect the bulk potential, but the difficulty consists in the determination of a solution for bulk images. However, the main contribution to the signal in our approach is a surface one, and it was determined with regard for the images. Therefore, the neglect of images for the bulk signal affects slightly the result. On the whole, the structure of the field $\mathbf{E}$ in the approach in (85) is calculated much more exactly. Moreover, the dependence of the signal on the resonator sizes is sensed and discovered. The consideration of images should not exclude, obviously, this dependence.

This reasoning implies that the correct calculation of the field in a resonator is a sufficiently complicated problem. In our opinion, formulas (84)-866) lead to a significantly more exact result, qualitatively and quantitatively, than linear relations of the form $\mathbf{E}=$ const $\times \mathbf{P}$.

\section{Appendix B. Polarizability of liquid ${ }^{4} \mathrm{He}$}

Of interest is the question about how the presence of a tidal DM of He II atoms is manifested in properties of $\varepsilon(T, \rho)$. The Clausius-Mossotti relation

$$
\varepsilon-1=\frac{4 \pi \rho}{3} \frac{A}{M}(\varepsilon+2)
$$


implies that $\varepsilon$ depends on $T$ via the density $\rho(T)$ and the polarizability $A(T)$. The dependence $A(T)$ was measured in Refs. 54, 55, where it was determined that $A$ decreases smoothly with increase in $T$ in the interval $T \approx 1.5 \div 2.7 \mathrm{~K}$, has a break at $T=T_{\lambda}$, and increases with $T$ in the interval $T \approx 2.7 \div 3.8 \mathrm{~K}$. In this case, at densities corresponding to $T \approx 1.5 \mathrm{~K} \div T_{\lambda}$ and $T \approx 2.7 \div 3.8 \mathrm{~K}$, $A$ decreases with increase in $\rho$. This fact is qualitatively explained in Refs. 56 .57 in the frameworks of two mechanisms which, however, do not take the mutual polarization of atoms into account. We note that $A$ increases with $\rho$ at $T \approx T_{\lambda} \div 2.7 \mathrm{~K}$, which remains unclear.

It was indicated in Ref. 58 that, at He II temperatures, $A$ depends on $T$ approximately as

$$
A \approx A_{0}\left(1+\delta_{0} / T\right)
$$

This relation corresponds to the Langevin-Debye law ${ }^{39}$

$$
\frac{\varepsilon-1}{\varepsilon+2}=\frac{4 \pi n d_{\mathrm{in}}^{2}}{9 k_{B} T}+A_{0} \frac{4 \pi \rho}{3 M}
$$

that is valid for a gas of molecules possessing the intrinsic DM $d_{\text {in }}$. Therefore, it was assumed in Ref. 58 that the dependence $A(T)$ (92) is related to the intrinsic DM of helium atoms. This gives the alternative explanation of the dependence $A(T)$ for $T \leq T_{\lambda}$.

The data $A(T=1.5 \mathrm{~K})=0.1232 \mathrm{~cm}^{3} /$ mole and $A(T=$ $2.0 \mathrm{~K})=0.12305 \mathrm{~cm}^{3} /$ mole (see Ref. 55) yield $A_{0} \approx$ $0.1226 \mathrm{~cm}^{3} /$ mole and $\delta_{0} \approx 9 \mathrm{~K} / 1226$. Since $\varepsilon=1.057195$ (see Ref. 54) and $\rho=0.14526 \mathrm{~g} / \mathrm{cm}^{3}$ at $T=1.63 \mathrm{~K}$ (see Ref. 55), relation (91) yields

$$
\varepsilon=\varepsilon_{0}\left(1+\frac{\beta_{0} \delta_{0}}{T}\right)+\frac{4 \pi A}{3 M}(\varepsilon+2)(\rho(T)-\rho(1.63 \mathrm{~K})),
$$

where $\varepsilon_{0} \approx 1.057$ and $\beta_{0} \approx 0.0539$. According to the modern theory, a free ${ }^{4} \mathrm{He}$ atom has no intrinsic DM. However, in the environment of other helium atoms, the atom acquires a fluctuating tidal DM with the mean modulus $\tilde{d}$. The shape of the function $g(r)$ (see Ref. 59) for He II testifies that the mean deviation of the interatomic distance in He II from $\bar{R}$ is about $\delta R \simeq \bar{R} / 6$. Let the distance from the given He II atom to the left adjacent atom be $\bar{R}-\delta R$, and let the distance to the right one be $\bar{R}$. We also mention another characteristic configuration with the distances $\bar{R}+\delta R$ and $\bar{R}$. According to (44) and (5), these neighbors induce the DM $d_{x}^{1}=d_{0}\left(\frac{\bar{R}^{7}}{(\bar{R}-\delta R)^{7}}-1\right)$ on the atom for the first configuration and $d_{x}^{2}=d_{0}\left(1-\frac{\bar{R}^{7}}{(\bar{R}+\delta R)^{7}}\right)$ for the second one, and the mean value is $d_{x} \simeq\left(d_{x}^{1}+d_{x}^{2}\right) / 2 \approx 1.6 d_{0}$. In the $Y$ and $Z$ directions, the induced DM of the atom is the same, so that the mean modulus of the total DM of the atom is $\tilde{d} \approx \sqrt{3} d_{x} \approx 2.8 d_{0}$. Such an estimate is analogous to the derivation of formula (17) in Ref. 7. The consideration of the structural factor $S(k)$ leads to formula $(30)$ in Ref. 7, where the coefficient is greater by a factor of 3.24. So, we should increase $\tilde{d}$ by approximately the same factor in order to take $S(k)$ into account. Finally, we get

$$
\tilde{d} \approx 2.8 \cdot 3.24 d_{0} \approx 3.22 \cdot 10^{-4}|e| a_{B} \approx 2.73 \cdot 10^{-33} \mathrm{C} \cdot \mathrm{m} .
$$

The quantity $\tilde{d}$ turns out to be greater than $\left|d_{\mathrm{h}}\right|$ (11): $\tilde{d} \approx-2.44 d_{\mathrm{h}}$. At the same time, relations (91)-93) yield $d_{\text {in }} \approx 3.2 \cdot 10^{-33} \mathrm{C} \cdot \mathrm{m} \approx 1.17 \tilde{d}$, i.e. $d_{\text {in }}$ is close to $\tilde{d}$.

We note that the DM vector of an atom averaged over the time is zero. But the mean DM modulus is nonzero, and the Langevin-Debye formula (93) is valid just for the nonzero DM modulus 60 .

For fluids, we should add the factor $q=(1-i \omega \tau)^{-1}$ (see Ref. 60) to the right-hand side of (93), where $\omega$ is the external field frequency, and $\tau=8 \pi \eta \tilde{a}^{3} /\left(k_{B} T\right)$ is the relaxation time. For He II, we obtain $\tau \sim 10^{-13} \mathrm{sec}^{-1}$ and $\omega \tau \sim 10$ (for experimental microwaves ${ }^{54}$ ). But this is true for polar molecules, whereas the DM of helium atoms is not intrinsic, but tidal. In the latter case, the value of $\tau$ is unknown, but it is probably much less due to the tough coupling with adjacent molecules, so that $q \approx 1$. We may conclude that formula (93) is valid at $\omega \tau \ll 1$ for liquid helium as well. Since $d_{\text {in }} \approx \tilde{d}$, it is possible that $q \approx 1$ for experimental values of $\omega$. Then the dependence $\varepsilon(T)$ at $T \leq T_{\lambda}$ can be explained by the tidal DM of He II atoms.

Because $\tilde{d} \sim \delta R / \bar{R}^{8} \sim \bar{R}^{-7} \sim \rho^{7 / 3}$ and $\delta_{0} \sim \tilde{d}^{2}$, we obtain that relation (92) can be written more exactly as

$$
A \approx A_{0}\left(1+\frac{\delta_{0} \cdot \rho^{14 / 3}(T)}{T \cdot \rho^{14 / 3}(1.63 \mathrm{~K})}\right) .
$$

Let us turn to the experimental dependence $A(T)$. It was assumed in Refs. 54,55 that, at the temperatures $T \approx 1.5 \mathrm{~K} \div T_{\lambda}$ and $T \approx 2.7 \div 3.8 \mathrm{~K}$, where $A$ decreases with increase in $\rho$, the dependencies $A(T)$ and $A(\rho)$ are determined $^{56,57}$ by a decrease in the distance between atoms with increase in $\rho$. In this case, the increase in $A$ with $\rho$ at $T \approx T_{\lambda} \div 2.7 \mathrm{~K}$ remains to be unclear. According to Ref. 58, the dependencies $A(T)$ and $A(\rho)$ on the interval $T \approx 1.5 \mathrm{~K} \div T_{\lambda}$ can be explained in a different way with the use of formulas (92) and (93). We consider that, for all $T$ and $\rho$, both mechanisms (a change in the density and the Langevin-Debye mechanism) contribute to the dependencies $A(T)$ and $A(\rho)$. In this case, the Langevin-Debye mechanism is related to the mutual polarization of atoms. If $q \approx 1$, then the main contribution to $A(T)$ at $T<2.7 \mathrm{~K}$ is given by the Langevin-Debye mechanism by (92) and (96). As $T$ increases, this contribution decreases and, at $T>2.7 \mathrm{~K}$, becomes less than the addition due to a change in the density. The latter is not taken into account in (92) and (96), but it determines the dependences $A(T)$ and $A(\rho)$ at $T>2.7 \mathrm{~K}$. It is easy to verify that formulas (92) and (96) well describe the experimental behavior of $A(T)$ and $A(\rho)$ (see Refs. 54,55) at all $T<2.7 \mathrm{~K}$, except for the break at $T=T_{\lambda}$. Thus, the behavior of $A(T)$ and $A(\rho)$ at $T \approx T_{\lambda} \div 2.7 \mathrm{~K}$, where $A$ increases with $\rho$, is explained as well. We note that it is difficult to determine which of formulas (92) or (96) corresponds better to the experiment ${ }^{54,55}$. If, nevertheless, 
$\omega \tau \gg 1$, then $q \ll 1$, and the Langevin-Debye mechanism leads to $A(T)$ much less than the experimental value and fails to describe the experimental $A(T)$ at $T<2.7 \mathrm{~K}$.
* Electronic address: mtomchenko@bitp.kiev.ua

1 A.S. Rybalko, Fiz. Nizk. Temp. 30, 1321 (2004) [Low Temp. Phys. 30, 994 (2004)].

${ }^{2}$ V.D. Khodusov, Vestn. Khark. Univ. N 642, 79 (2004).

3 A.M. Kosevich, Fiz. Nizk. Temp. 31, 50, 1100 (2005) [Low Temp. Phys. 31, 37, 920 (2005)].

${ }^{4}$ L.A. Melnikovsky, J. Low Temp. Phys. 148, 559 (2007).

5 E.A. Pashitsky, S.M. Ryabchenko, Fiz. Nizk. Temp. 33, 12 (2007) [Low Temp. Phys. 33, 8 (2007)].

6 V.M. Loktev, M.D. Tomchenko, Fiz. Nizk. Temp. 34, 337 (2008) [Low Temp. Phys. 34, 262 (2008)].

7 M.D. Tomchenko, J. Low Temp. Phys. 158, 854 (2010).

8 E.D. Gutlyanskii, Fiz. Nizk. Temp. 35, 956 (2009) [Low Temp. Phys. 35, 748 (2009)].

9 S.I. Shevchenko, A.S. Rukin, Pis'ma Zh. Eksp. Teor. Fiz. [JETP Lett.] 90, 46 (2009).

10 S.I. Shevchenko, A.S. Rukin, Fiz. Nizk. Temp. 36, 186 (2010) [Low Temp. Phys. 36, 146 (2010)].

11 E.A. Pashitsky, A.A. Gurin, Zh. Eksp. Teor. Fiz. 138, 1103 (2010) [JETP 111, 975 (2010)].

12 V.P. Mineev, J. Low Temp. Phys. 162, 686 (2011).

13 G.E. Volovik, Pis'ma Zh. Eksp. Teor. Fiz. 39, 169 (1984) [JETP Lett. 39, 200 (1984)].

14 W. Byers Brown, D.M. Whisnant, Mol. Phys. 25, 1385 (1973).

15 D.M. Whisnant, W. Byers Brown, ibid. 26, 1105 (1973).

16 V.M. Loktev and M.D. Tomchenko, J. Phys. B: At. Mol. Opt. Phys. 44, 035006 (2011); Rep. Nat. Acad. Sci. Ukr., N 5, 76 (2010) (in Russian).

17 M.D. Tomchenko, Phys. Rev. B 83, 094512 (2011).

18 M.D. Tomchenko, Rep. Nat. Acad. Sci. Ukr., N 1, 64 (2011) (in Russian).

19 M.D. Tomchenko, Ukr. J. Phys., 56, 144 (2011).

20 B. Linder, R.A. Kromhout, Phys. Rev. B 13, 1532 (1976).

21 L.W. Bruch and T. Osawa, Mol. Phys. 40, 491 (1980).

22 G. Herzberg, Atomic Spectra and Atomic Structure, Dover, New York (1944).

23 T. Kinoshita, Phys. Rev. 115, 366 (1959).

24 E. Cheng, M.W. Cole, W.F. Saam, J. Treiner, Phys. Rev. B 46, 13967 (1992).

25 E. Cheng, M.W. Cole, J. Dupont-Roc, W.F. Saam, J. Treiner, Rev. Mod. Phys. 65, 557 (1993).

26 E. Zaremba, W. Kohn, Phys. Rev. B 15, 1769 (1977).

27 V.L. Ginzburg, Zh. Eksp. Teor. Fiz. [JETP] 29, 254 (1955).

${ }^{28}$ G.A. Gamtsemlidze, ibid. 34, 1434 (1958).

29 V.L. Ginzburg and L.P. Pitaevskii, ibid. 34, 1240 (1958).

30 J.H. Scholtz, E.O. McLean and I. Rudnick, Phys. Rev. Lett. 32, 147 (1974).

31 S. Putterman, Superfluidity Hydrodynamics, NorthHolland, Amsterdam (1974), Chap. 5.

32 G.W. Rayfield and F. Reif, Phys. Rev. 136, A1194 (1964).

33 V. Apaja and E. Krotscheck, Phys. Rev. B 67, 184304
(2003).

34 V. Apaja and E. Krotscheck, J. Low Temp. Phys. 134, 793 (2004).

35 O. Plantevin, B. Fak, H.R. Glyde et al., Phys. Rev. B 63, 224508 (2001).

36 P.L. Kapitsa, Zh. Eksp. Teor. Fiz. [JETP] 11, 1 (1941).

37 I. M. Khalatnikov, ibid. 22, 687 (1952).

38 B.N. Esel'son, V.N. Grigor'ev, V.G. Ivantsov, E.Ya. Rudavskii, Properties of Liquid and Solid Helium, Izd. Standartov, Moscow (1978) (in Russian).

39 I.E. Tamm, Foundations of Electricity Theory, Fizmatlit, Moscow (2003) (in Russian).

40 S.I. Novikova, Thermal Expansion of Solids, Nauka, Moscow (1974), Chap. 2 (in Russian).

41 A.S. Davydov, Solid State Physics, Acad. Press, New York (1965), Chap. 4.

42 A. Chizmeshya, M.W. Cole, E. Zaremba, W. Kohn, J. Low Temp. Phys. 110, 677 (1998).

43 P.J. Nacher and J. Dupont-Roc, Phys. Rev. Lett. 67, 2966 (1991).

44 I.M. Khalatnikov, An Introduction to the Theory of Superfluidity, Perseus, Cambridge (2000).

45 S.I. Vil'chinsky, E.A. Pashitsky, P.I. Fomin, Fiz. Nizk. Temp. 23, 1267 (1997) [Low Temp. Phys. 23, 951 (1997)].

46 A.R. Hippel, Dielectrics and waves, John Wiley and Sons, N. Y. (1954).

47 A.S. Rybalko, private communication.

48 M.R. Gibbs, K.H. Andersen, W.G. Stirling, H. Schober, J. Phys. Cond. Mat. 11, 603 (1999).

49 V.P. Peshkov, Zh. Eksp. Teor. Fiz. [JETP] 18, 867 (1948).

50 Physical Encyclopedia, Sovet. Entsiklopediya, Moscow (1990), (in Russian), vol. 2, p. 241.

51 V.P. Peshkov, Zh. Eksp. Teor. Fiz. [JETP] 18, 857 (1948).

52 R.P. Henkel, E.N. Smith and J.D. Reppy, Phys. Rev. Lett. 23, 1276 (1969).

53 L.D. Landau, E.M. Lifshitz, Electrodynamics of Continuous Media, Pergamon, New York (1984).

54 J. Stankovski, S. Sitarz, Z. Trybula, W. Kempinski, T. Zuk, Acta Phys. Polonica A 70, 291 (1986).

${ }^{55}$ W. Kempinski, T. Zuk, J. Stankovski and S. Sitarz, Fiz. Nizk. Temp. 14, 451 (1988).

56 C.A. Ten Seldam and S.R. De Groot, Physica 18, 905 (1952).

57 P. Mazur and L. Jansen, Physica 21, 208 (1955).

58 A.S. Rybalko, S.P. Rubets, E.Ya. Rudavskii, V.A. Tikhiy, R. Golovashchenko, V.N. Derkach, S.I. Tarapov, arXiv:cond-mat/0807.4810 (2008).

59 E.C. Svensson, V.F. Sears, A.D.B. Woods and P. Martel, Phys. Rev. B 21, 3638 (1980).

60 P. Debye, Selected Works, Nauka, Leningrad (1987), (in Russian), Chap. 2. 